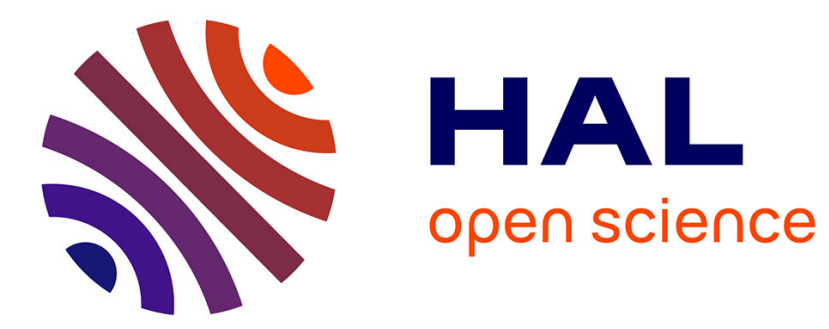

\title{
Sur les Théorèmes I et II de Painlevé
}

\author{
Frank Loray
}

\section{To cite this version:}

Frank Loray. Sur les Théorèmes I et II de Painlevé. Geometry and Dynamics, International conference in honnour of the 60th Anniversary of Alberto Verjovsky, 2005, Cuernavaca, Mexico. pp.165-190. hal-00001191

\section{HAL Id: hal-00001191 \\ https://hal.science/hal-00001191}

Submitted on 2 Mar 2004

HAL is a multi-disciplinary open access archive for the deposit and dissemination of scientific research documents, whether they are published or not. The documents may come from teaching and research institutions in France or abroad, or from public or private research centers.
L'archive ouverte pluridisciplinaire HAL, est destinée au dépôt et à la diffusion de documents scientifiques de niveau recherche, publiés ou non, émanant des établissements d'enseignement et de recherche français ou étrangers, des laboratoires publics ou privés. 


\title{
SUR LES THÉORÈMES I ET II DE PAINLEVÉ
}

\author{
par \\ Frank LORAY
}

en l'honneur d'Alberto Verjovsky

\begin{abstract}
Résumé. - Un siècle avant Alberto, Paul Painlevé était lui aussi professeur à l'Université de Lille et posait les fondations des notions de feuilletage et d'holonomie à travers deux théorèmes que l'on trouve aujourd'hui au début des "Leçons de Stockholm". Je remercie Alberto de m'avoir encouragé à les lire et je propose ici d'en donner un énoncé précis ainsi qu'une preuve rigoureuse illustrée par de nombreux exemples. Je termine par deux conjectures quant au comportement global des applications d'holonomie, localement définies par le Théorème II.
\end{abstract}

\section{Introduction}

Paul Painlevé a implicitement introduit la notion de feuilletage holomorphe singulier dans la première partie de ses "Leçons de Stockholm" lorsqu'il étudie les propriétés des équations différentielles algébriques du premier ordre $F\left(y^{\prime}, y, x\right)=0$ (spécialement dans les "Leçons 2 et 3").

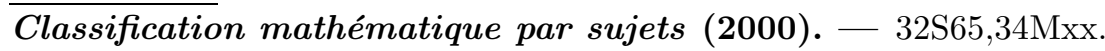

Mots clefs. - Équations différentielles dans le domaine complexe, Points singuliers mobiles, Prolongement analytique et Singularités. 
Afin de faciliter l'exposé, nous ne discuterons que des équations du premier degré :

$$
\text { (E) } \quad \frac{d y}{d x}=\frac{P(x, y)}{Q(x, y)}
$$

avec $P, Q \in \mathbb{C}[x, y]$ polynomiaux dans chacune des variables $(x, y) \in \mathbb{C}^{2}$. Si les conditions initiales $\left(x_{0}, y_{0}\right) \in \mathbb{C}^{2}$ satisfont $Q\left(x_{0}, y_{0}\right) \neq 0$, alors le théorème de Cauchy implique l'existence d'une unique solution $y=$ $f(x)$ (pour l'équation $(E)$ ) qui est analytique sur un voisinage $D$ de $x_{0}$ et telle que $f\left(x_{0}\right)=y_{0}$. Nous noterons $f\left(x, x_{0}, y_{0}\right)$ cette solution. Ce théorème est purement local. Que peut-on dire des solutions globales ? Plus précisément, que peut-on dire du prolongement analytique et des singularités possibles de $f\left(x, x_{0}, y_{0}\right)$ dans la variable $x$ ? ou encore $y_{0}$ ?

\section{Prolongement analytique et fonctions multiformes}

Rappelons les notions de prolongement analytique et de surface de Riemann d'une fonction multiforme. Dans toute la suite, $\Omega$ désigne un domaine (connexe) de $\mathbb{C}, f$ un germe de fonction holomorphe en un point $x_{0} \in \Omega, \gamma:[0,1] \rightarrow \Omega$ un chemin (continu ou différentiable) issu de $\gamma(0)=x_{0}$ et $x_{1}:=\gamma(1)$ son extrémité.

Définition 1.1. - On dit qu'une suite de disques $D_{0}, D_{1}, \ldots, D_{n} \subset \Omega$ recouvre $\gamma$ si, pour un découpage convenable $0=t_{0}<t_{1}<\cdots<t_{n}<$ $t_{n+1}=1$ de l'intervalle, $D_{k}$ contient $\gamma\left(\left[t_{k}, t_{k+1}\right]\right)$ pour $k=0, \ldots, n$. On dit que $f$ admet un prolongement analytique le long de $\gamma$ s'il existe une suite de disque $D_{0}, D_{1}, \ldots, D_{n} \subset \Omega$ recouvrant $\gamma$ et des fonctions holomorphes $f_{k}: D_{k} \rightarrow \mathbb{C}$ telles que $f_{0} \equiv f$ au voisinage de $x_{0}$ et $f_{k} \equiv f_{k-1}$ sur $D_{k-1} \cap D_{k}$ pour tout $k=1, \ldots, n$. Le germe de fonction défini par $f_{n}$ en $x_{1}:=\gamma(1)$ sera noté $f_{\gamma}$ et appelé détermination de $f$ au dessus de $x_{1}$.

Remarque 1.2. - Dans la définition précédente, la détermination $f_{\gamma}$ ne dépend que de la chaine de disques $\left(D_{k}\right)_{k}$. En particulier, pour $\varepsilon>0$ suffisamment petit, $f$ admet un prolongement analytique le long de toute $\varepsilon$-perturbation de $\gamma$, c'est à dire de tout chemin $\gamma^{\prime}:[0,1] \rightarrow \Omega$ satisfaisant $\left|\gamma^{\prime}(t)-\gamma(t)\right|<\varepsilon$ et $\gamma^{\prime}(0)=x_{0}$. De plus, dès que $\gamma^{\prime}(1)=x_{1}$, ce nouveau chemin nous conduit à la même détermination $f_{\gamma^{\prime}} \equiv f_{\gamma}$. 


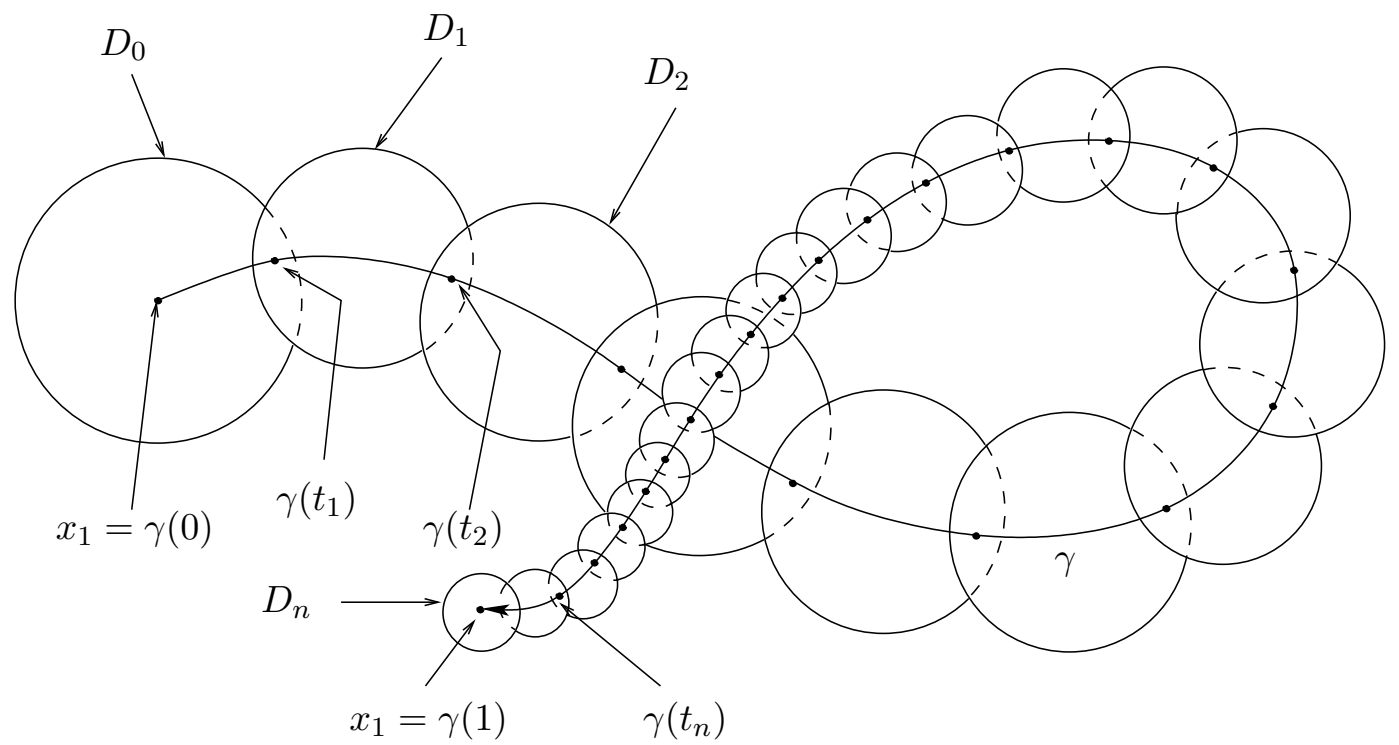

Figure 1. Prolongement analytique

Ainsi, quitte à remplacer $\gamma$ par une perturbation, on pourra toujours supposer $\gamma$ différentiable dans la définition précédente et celles qui suivent.

Lemme (Poincaré-Volterra). - Au dessus de chaque point $x_{1} \in \Omega$, l'ensemble :

$$
\left\{f_{\gamma} ; \gamma:[0,1] \rightarrow \Omega, \gamma(0)=x_{0} \text { et } \gamma(1)=x_{1}\right\}
$$

des déterminations de $f$ est au plus dénombrable.

Démonstration. - Toute détermination $f_{\gamma}$ est déterminée par la suite de disques $\left(D_{k}\right)_{k}$ recouvrant $\gamma$. Quitte à remplacer les $D_{k}$ par des disques très proches (ce qui ne modifie pas le germe de fonction obtenu en $x_{1}$ ) on peut supposer leur rayon et les coordonnées de leur centre rationnels. L'ensemble des suites finies de disques à coordonnées rationnelles étant dénombrable, l'ensemble des déterminations l'est aussi. 
Proposition 1.3. - Il existe une surface de Riemann (connexe) $\mathcal{S}$ avec un point marqué $p_{0} \in \mathcal{S}$ et une application holomorphe $\phi: \mathcal{S} \rightarrow \Omega \times \mathbb{C}$, $\phi=(\pi, \widetilde{f})$, satisfaisant :

1. $\pi\left(p_{0}\right)=x_{0}$ et $f \circ \pi \equiv \widetilde{f}$ au voisinage de $p_{0}$;

2. $\pi: \mathcal{S} \rightarrow \Omega$ est un difféomorphisme local en tout point de $\mathcal{S}$;

3. tout autre triplet $\left(\mathcal{S}^{\prime}, p_{0}^{\prime}, \phi^{\prime}\right)$ satisfaisant (1) et (2) se factorise par $\left(\mathcal{S}, p_{0}, \phi\right)$ via une application holomorphe $\varphi: \mathcal{S}^{\prime} \rightarrow \mathcal{S}: \varphi\left(p_{0}^{\prime}\right)=p_{0}$ et $\phi^{\prime}=\phi \circ \varphi$.

Le triplet $\left(\mathcal{S}, p_{0}, \phi\right)$ est unique à isomorphisme près. Le graphe $\mathcal{G}:=\phi(\mathcal{S})$ est unique.

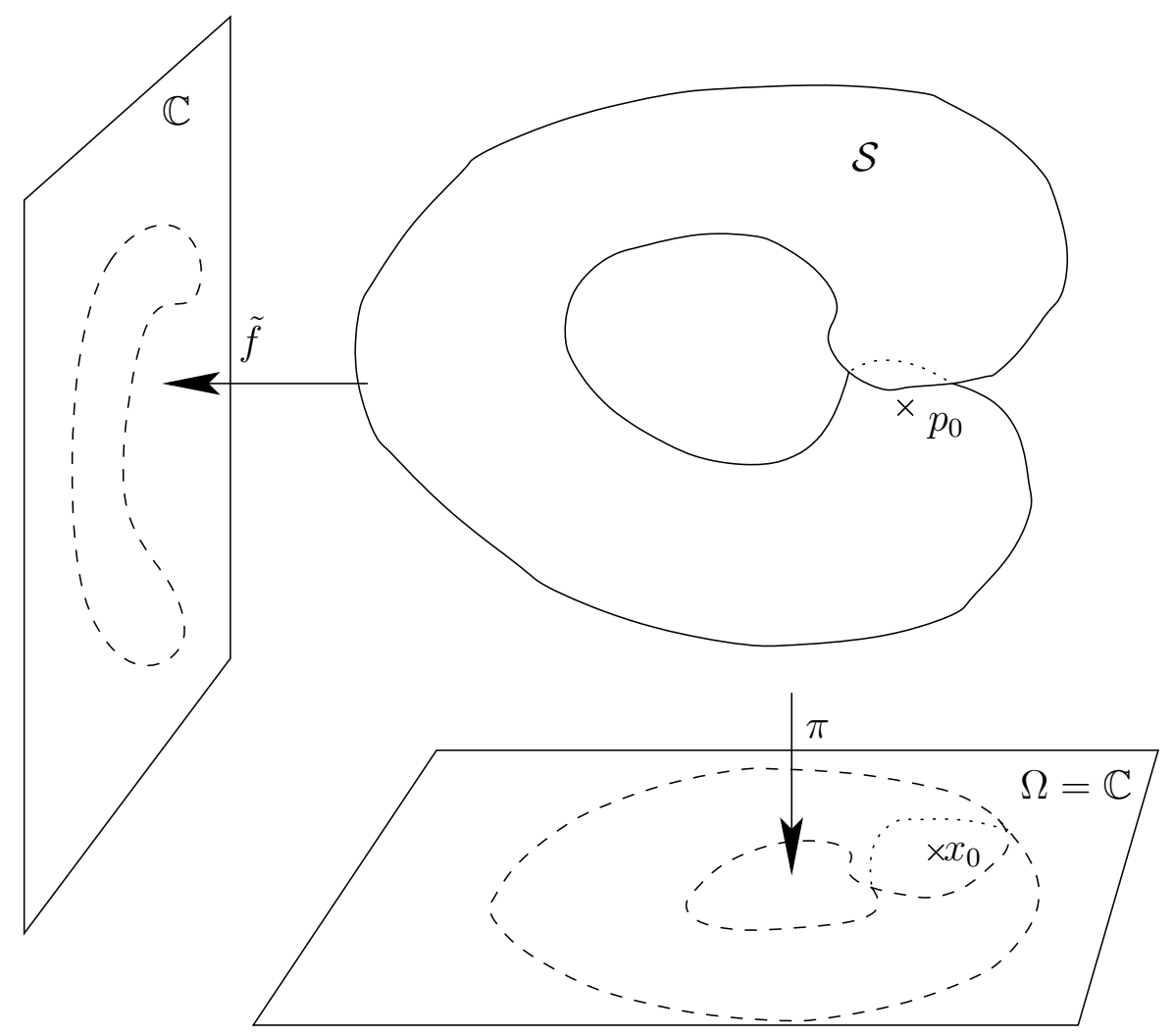

Figure 2. Surface de Riemann (ou graphe) de $f$ 
Idée de preuve (voir 10 ). - La surface est construite comme l'ensemble des couples $\left(\gamma(1), f_{\gamma}\right)$ où $\gamma$ décrit l'ensemble des chemins $\gamma:[0,1] \rightarrow \Omega$ issus de $\gamma(0):=x_{0}$ le long desquels $f$ admet un prolongement analytique. Si $D$ est le disque de convergence de $f_{\gamma}$, alors l'ensemble des couples $\left(\gamma^{\prime}(1), f_{\gamma^{\prime}}\right)$ obtenus par concaténations $\gamma^{\prime}=\gamma^{\prime \prime} \cdot \gamma$ avec les chemins $\gamma^{\prime \prime}:[0,1] \rightarrow D$ issus de $\gamma^{\prime \prime}(0):=x_{1}$ forme un voisinage $V\left(p_{1}\right)$ du point $p_{1}:=\left(x_{1}, f_{\gamma}\right)$ de la surface. Par Poincaré-Volterra, une collection dénombrable de telles cartes suffit pour recouvrir la surface. L'application $\phi$ est alors définie sur $V\left(p_{1}\right)$ par la flèche $\left(x_{1}^{\prime}, f_{\gamma^{\prime}}\right) \mapsto\left(x_{1}^{\prime}, f_{\gamma^{\prime}}\left(x_{1}^{\prime}\right)\right)$.

Remarque 1.4. - Le germe $f$ admet un prolongement analytique le long de $\gamma$ si et seulement si $\gamma$ se relève en $\widetilde{\gamma}:[0,1] \rightarrow \mathcal{S}$ satisfaisant $\widetilde{\gamma}(0)=p_{0}$ et $\gamma \equiv \pi \circ \widetilde{\gamma}$. Alors, $f_{\gamma}$ est le germe de fonction défini en $\gamma(1)$ par l'égalité $f_{\gamma} \circ \pi=\tilde{f}$ en $\widetilde{\gamma}(1)$.

Définition 1.5. - On appellera fonction multiforme définie sur $\Omega$ par le germe $f$ le "prolongement analytique maximal" de $f$ sur $\Omega$ donné ou bien par sa surface de Riemann $\phi: \mathcal{S} \rightarrow \Omega \times \mathbb{C}$ (à isomorphisme près), ou bien par son graphe $\mathcal{G}$. On notera $\underline{f}_{\Omega}$ cette "fonction" et, si nécessaire, $\phi_{\underline{f}_{\Omega}}: \mathcal{S}_{\underline{f}_{\Omega}} \rightarrow \Omega \times \mathbb{C}$ et $\mathcal{G}_{\underline{f}_{\Omega}}$.

Remarque 1.6. - La restriction d'une fonction multiforme sur $\Omega$ à un domaine $\Omega^{\prime} \subset \Omega$ n'a pas de sens : la préimage $\phi_{\underline{f}_{\Omega}}^{-1}\left(\Omega^{\prime} \times \mathbb{C}\right)$ peut avoir plusieurs composantes connexes dans $\mathcal{S}_{\underline{f}_{\Omega}}$ et définir autant de fonctions multiformes sur $\Omega^{\prime}$. Par contre, si $x_{0} \in \Omega^{\prime}$, alors la composante connexe contenant $p_{0}$ s'identifie à $\mathcal{S}_{\underline{f}_{\Omega^{\prime}}}$.

\section{Singularités}

Définition 2.1. - On dit que $\gamma$ conduit $f$ vers une singularité lorsque $f$ admet un prolongement analytique le long de $\left.\gamma\right|_{[0,1-\varepsilon]}$ pour tout $\varepsilon>0$ mais pas le long de $\gamma$. On dit qu'un autre chemin $\gamma^{\prime}:[0,1] \rightarrow \Omega$ joignant $x_{0}$ à $x_{1}$ conduit $f$ vers la même singularité si pour tout voisinage $D$ de $x_{1}$, les déterminations $f_{\left.\gamma\right|_{[0, \tau]}}$ et $f_{\gamma^{\prime} \mid[0, \tau]}$ définissent la même fonction multiforme sur $D$ pour $\tau$ suffisamment proche de 1 . 
On appelle singularité de $f$ au dessus de $\Omega$ (ou de $\underline{f}_{\Omega}$ ) définie par $\gamma$ la classe d'équivalence notée $f_{\gamma}$ pour la relation précédente. On note $\widetilde{\Sigma}_{\underline{f}_{\Omega}}$ (resp. $\Sigma_{\underline{f}_{\Omega}}$ ) l'ensemble des singularités $f$ au dessus de $\Omega$ (resp. de leur projection sur $\Omega$ ).

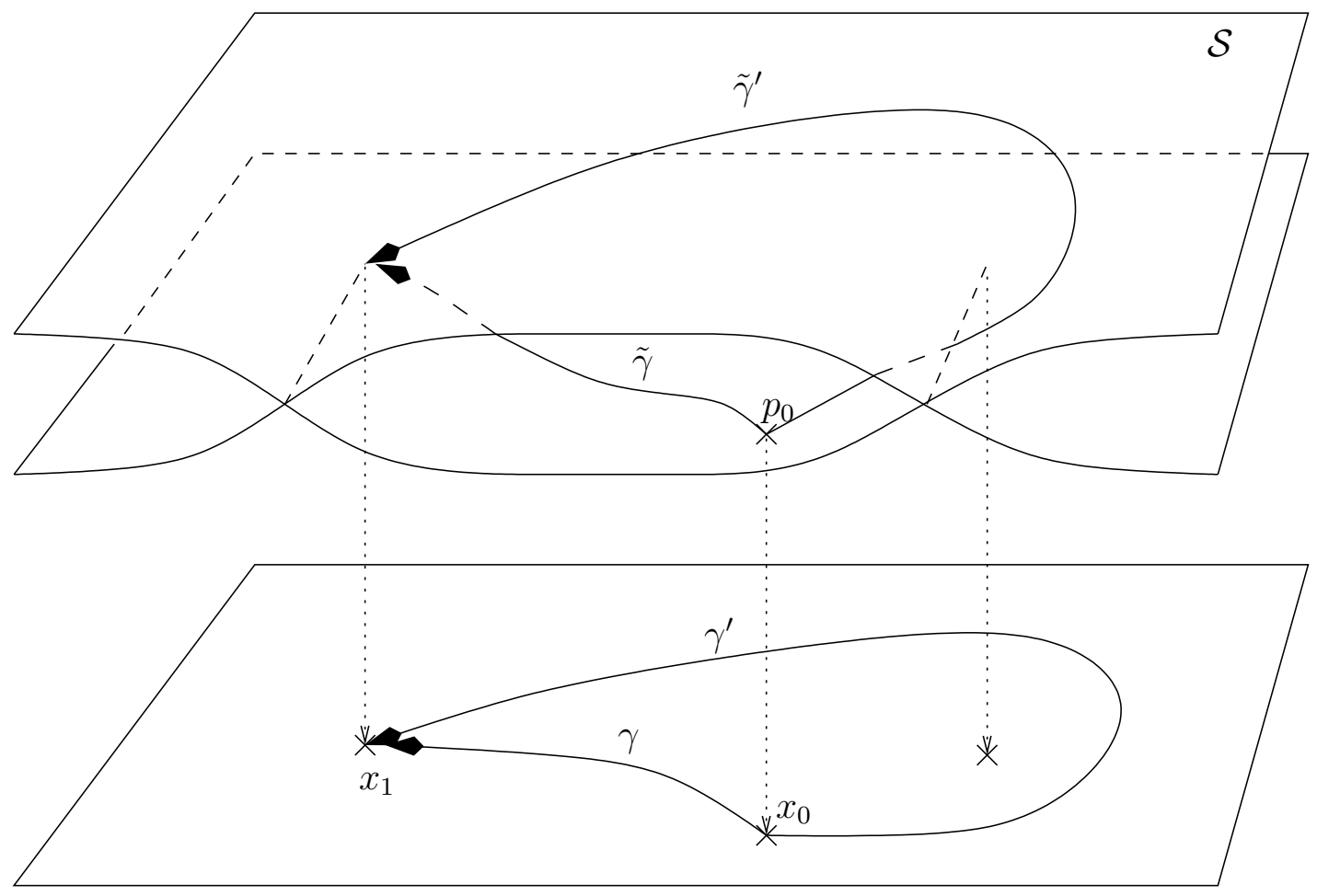

Figure 3. Deux chemins conduisant vers une même singularité

Remarque 2.2. - En fait, $\gamma$ conduit $f$ vers une singularité si et seulement si le chemin incomplet $\left.\gamma\right|_{[0,1[}:[0,1[\rightarrow \Omega$ se relève, via la projection $\pi: \mathcal{S} \rightarrow \Omega$, en un chemin propre $\widetilde{\gamma}:[0,1[\rightarrow \mathcal{S}$ (c'est à dire $\widetilde{\gamma}(t)$ tend vers le bord de $\mathcal{S}$ lorsque $t \rightarrow 1$ ). 
Définition 2.3. - On dit que $f$ définit une fonction multiforme régulière sur $\Omega$ si $f$ ne possède pas de singularité au dessus de $\Omega$, i.e. admet un prolongement analytique le long de tout chemin $\gamma:[0,1] \rightarrow \Omega$ issu de $\gamma(0)=x_{0}$. C'est le cas si et seulement si l'application $\pi: \mathcal{S} \rightarrow \Omega$ est un revêtement. En particulier, le nombre de déterminations de $f$ au dessus d'un point $x_{1} \in \Omega$ est independant de $x_{1}$; si $\Omega$ est simplement connexe, alors l'extension est uniforme.

Exemple 2.4. - La détermination principale du logarithme $f(x)=$ $\log (x)$ en $x_{0}=1, f(1)=0$, définit une fonction uniforme sur $\mathbb{C} \backslash \mathbb{R}^{-}$, multiforme régulière sur $\mathbb{C}^{*}$ et possède, au dessus de $\mathbb{C}$, une unique singularité $\gamma$ qui se projette sur 0 .

Exemple 2.5. - La fonction multiforme définie par $f(x)=x^{\alpha}:=$ $\exp (\alpha \log (x)), \alpha \in \mathbb{C}^{*}$, solution de l'équation différentielle $y^{\prime}=\alpha \frac{y}{x}$, possède exactement une singularité au dessus de 0 excepté lorsque $\alpha \in \mathbb{N}$. Elle possède une infinité de déterminations au dessus de chaque point autre que 0 sauf quand $\alpha$ est rationnel : si $\alpha=\frac{p}{q} \in \mathbb{Q}$, alors $f$ possède exactement $q$ déterminations. Enfin, $f$ admet une limite en 0 si et seulement si $\alpha$ est réel : $f(x)$ tend vers 0 si $\alpha>0$ et $\infty$ si $\alpha<0$ dès que $x \rightarrow 0$ le long de n'importe quel chemin $\gamma$ représentant la singularité.

Exemple 2.6. - La fonction $f(x)=\alpha_{1} \log \left(x-\zeta_{1}\right)+\alpha_{2} \log \left(x-\zeta_{2}\right)+$ $\alpha_{3} \log \left(x-\zeta_{3}\right)$, solution de l'équation différentielle $\frac{d y}{d x}=\frac{\alpha_{1}}{x-\zeta_{1}}+\frac{\alpha_{2}}{x-\zeta_{2}}+\frac{\alpha_{3}}{x-\zeta_{3}}$, est régulière sur $\mathbb{C} \backslash\left\{\zeta_{1}, \zeta_{2}, \zeta_{3}\right\}$. Au dessus d'un point distinct des $\zeta_{k}$, deux déterminations diffèrent d'une constante appartenant au groupe additif $G=2 \pi i\left(\alpha_{1} \mathbb{Z}+\alpha_{2} \mathbb{Z}+\alpha_{3} \mathbb{Z}\right)$. Au dessus de $\zeta_{1}$, deux singularités diffèrent d'une constante appartenant au sous-groupe $H_{1}=2 \pi i\left(\alpha_{2} \mathbb{Z}+\right.$ $\left.\alpha_{3} \mathbb{Z}\right)$. La fonction inverse $x=g(y)$, solution de l'équation différentielle $\frac{d x}{d y}=1 /\left(\frac{\alpha_{1}}{x-\zeta_{1}}+\frac{\alpha_{2}}{x-\zeta_{2}}+\frac{\alpha_{3}}{x-\zeta_{3}}\right)$, possède au moins comme singularités les valeurs critiques de $f$. En général, la dérivée $f^{\prime}(x)$ s'annule au dessus de deux points $x_{1} \neq x_{2}$ distincts des $\zeta_{k}$ et pour toutes les détermination. Pour des $\alpha_{k}$ génériques, le groupe $G$ est dense dans $\mathbb{C}$; par suite, les singularités de $g$ se projettent sur un sous-ensemble $\Sigma$ dense de $\mathbb{C}$. 


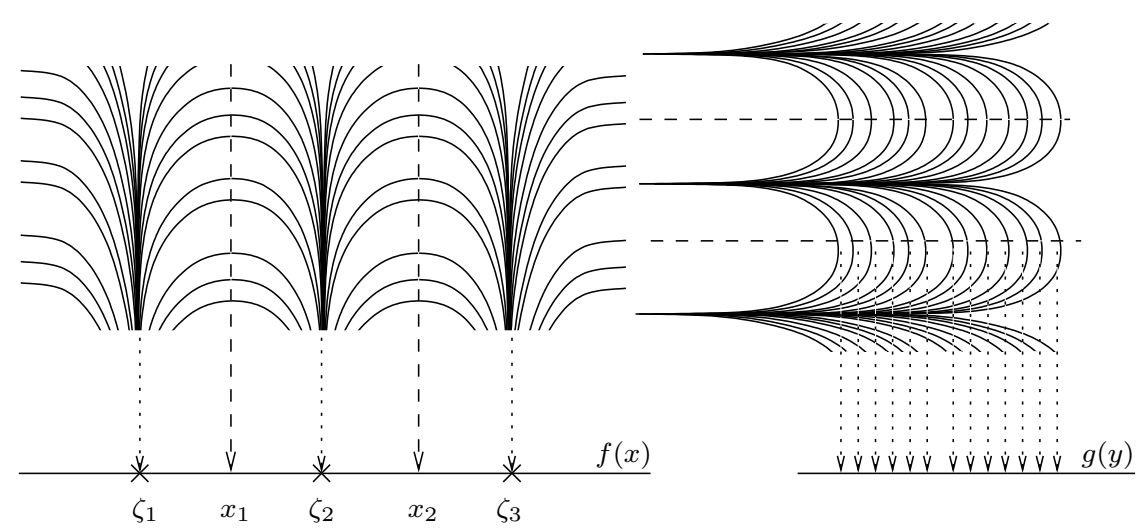

Figure 4. Exemple 2.6

Exemple 2.7. - La fonction multiforme définie sur $\mathbb{C}$ par $f(x)=$ $\sqrt{x^{\alpha}-1}, \alpha=\frac{\log (2)}{2 \pi i}$, solution de l'équation différentielle $y^{\prime}=\frac{\alpha\left(y^{2}+1\right)}{2 x y}$, possède exactement une singularité au dessus de chaque point $x_{n}=2^{n}$ et au dessus de 0 . Précisément, sur $\mathbb{C} \backslash \mathbb{R}^{-}$et pour la détermination principale de $x^{\alpha}=\exp (\alpha \log (x)), f$ possède exactement une singularité au dessus de $x_{0}=1$ et 2 déterminations autour : notons $f^{+}$et $f^{-}$les fonctions uniformes induites sur $\mathbb{C} \backslash]-\infty, 0] \cup[1,+\infty[$. Après avoir tourné $n$ fois autour de $0, n \in \mathbb{Z}$, la situation est essentiellement la même au dessus de $\mathbb{C} \backslash \mathbb{R}^{-}$: l'unique singularité est maintenant au dessus $x_{n}=2^{n}$ et les 2 nouvelles déterminations uniformes sur $\mathbb{C} \backslash]-\infty, 0] \cup\left[x_{n},+\infty[\right.$ respectivement notées $f_{n}^{+}$et $f_{n}^{-}$se permuttent autour de $x_{n}$. Ainsi, au dessus de chacun des $x_{n}$, il y a exactement une singularité et une infinité de déterminations régulières. Au dessus de 0 , tous les chemins mênent à la même singularité : à l'intérieur de tout disque $D$ centré en 0 , toutes les déterminations $f_{n}^{+}$et $f_{n}^{-}$(en un point générique) s'échangent par prolongement analytique dans $D$. En effet, on passe de $f_{n}^{+}$à $f_{n+1}^{+}$(resp. de $f_{n}^{-}$à $f_{n+1}^{-}$) en tournant autour de 0 ; enfin, pour $n>>0, x_{n}$ est dans $D$ et on passe de $f_{n}^{+}$à $f_{n}^{-}$en tournant autour. Cette singularité est plus compliquée que les précédentes car d'autres singularités l'accumulent. 


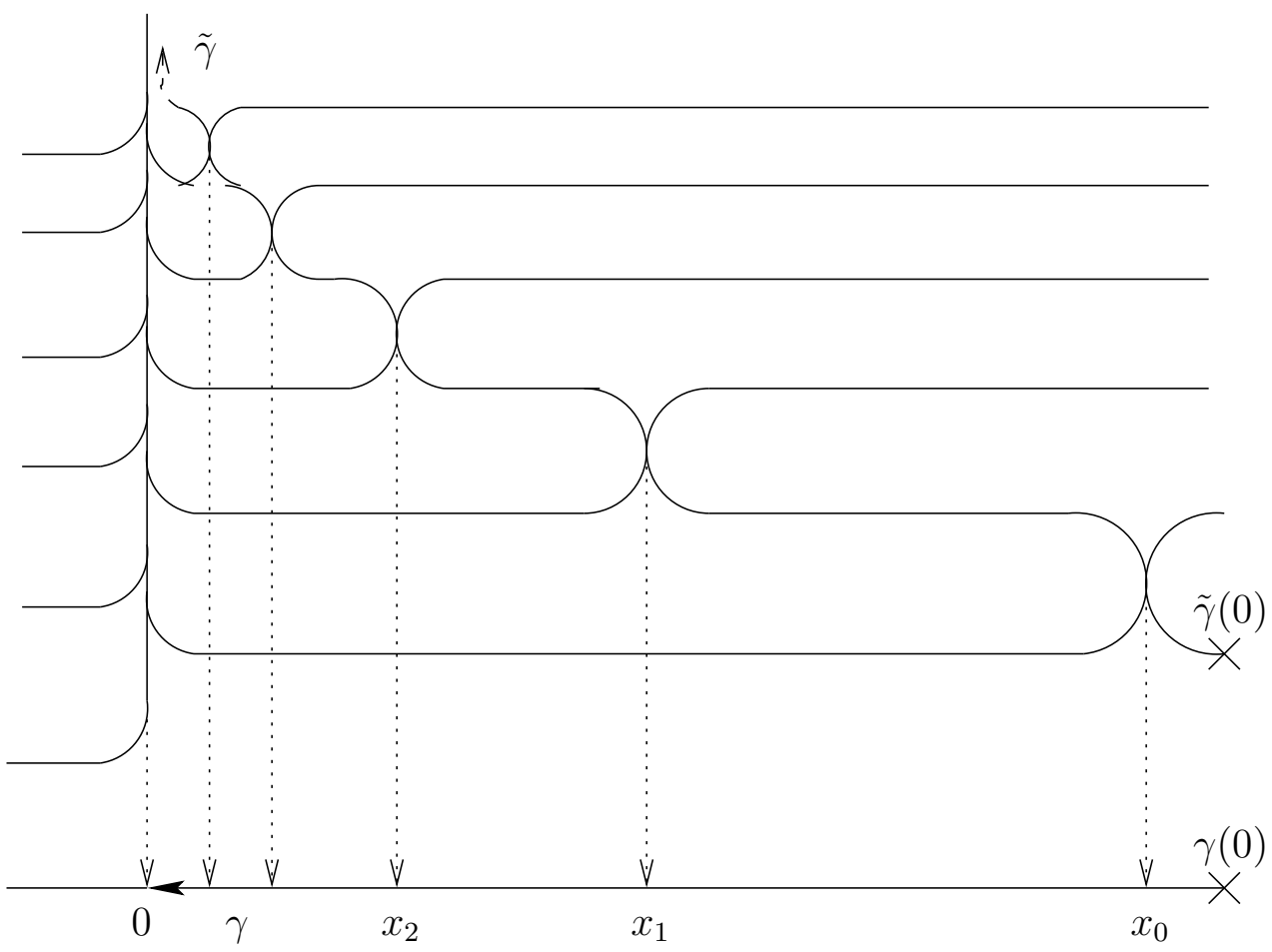

Figure 5. La singularité non isolée $\tilde{\gamma}$ de l'exemple 2.7

Exemple 2.8. - Le revêtement universel $\{|x|<1\} \rightarrow \mathbb{C} \backslash\{0,1\}$ est une fonction uniforme avec frontière naturelle : il n'existe pas de prolongement analytique au delà du cercle $\Sigma=\{|x|=1\}$.

Les différents types de singularités apparaissant dans les exemples précédents se distinguent modulo quelques définitions supplémentaires.

Définition 2.9. - Une singularité $f_{\gamma}$ est dite isolée s'il existe un disque $D$ centré en $x_{1}:=\gamma(1)$ tel que la fonction multiforme ${\underline{f_{\gamma}}}_{D}$ qu'elle définit sur $D$ soit régulière sur $D^{*}:=D \backslash\left\{x_{1}\right\}$.

Remarque 2.10. - La singularité définie par $\gamma$ est isolée si et seulement si il existe $\varepsilon>0$ tel que $f$ admette un prolongement analytique le long de tout chemin $\gamma^{\prime}:[0,1] \rightarrow \Omega$ issu de $x_{0}$, $\varepsilon$-proche de $\gamma$ évitant $x_{1}$ :

$$
\left.\left.\left|\gamma^{\prime}(t)-\gamma(t)\right|<\varepsilon \quad \text { et } \quad x_{1} \notin \gamma^{\prime}(] 0,1\right]\right) \text {. }
$$


Définition 2.11. - On dit que $f$ admet $y_{1} \in \overline{\mathbb{C}}$ comme limite en une singularité $f_{\gamma}, \overline{\mathbb{C}}=\mathbb{C} \cup \infty$, si pour tout chemin $\gamma^{\prime}$ représentant cette singularité on a $\lim _{t \rightarrow 1} f\left(\gamma^{\prime}(1)\right)=y_{1}$.

Une singularité $f_{\gamma}$ est dite algébrö̈de si elle est isolée, possède un nombre fini de déterminations autour de $x_{1}:=\gamma(1)$ et possède une limite (finie ou infinie) en $x_{1}$.

Remarque 2.12. - Soit $f_{\gamma}$ une singularité isolée. Sont équivalents :

1. $f_{\gamma}$ possède un nombre fini de déterminations autour de $x_{1}=\gamma(1)$,

2. le relevé $\widetilde{\gamma}:[0,1[\rightarrow \mathcal{S}$ de $\gamma$, tend vers une composante parabolique du bord de $\mathcal{S}$ (i.e. vers un point $p_{1}$ dans une surface de Riemann $\left.\widehat{\mathcal{S}}=\mathcal{S} \cup\left\{p_{1}\right\}\right)$,

3. le graphe de la fonction multiforme $f_{\gamma}$ sur un disque $D$ suffisamment petit centré en $x_{1}$ est un disque épointé proprement plongé dans $D^{*} \times \mathbb{C}$,

4. le germe de fonction multiforme $f_{\gamma}$ est de la forme $g\left(\left(x-x_{1}\right)^{1 / q}\right)$ où $g$ est une fonction holomorphe sur un voisinage épointé de $0 \in \mathbb{C}$ et $q \in \mathbb{N}^{*}$.

On dit alors que la singularité est à monodromie finie et sont équivalents :

1. $f_{\gamma}$ est algébroïde,

2. $\widetilde{f}$ s'étend méromorphiquement en $p_{1}$,

3. $\widetilde{D^{*}}$ est la restriction à $D^{*} \times \mathbb{C}$ d'un disque $\widetilde{D} \subset D \times \overline{\mathbb{C}}$ proprement plongé,

4. $g$ s'étend méromorphiquement en 0 .

Les singularités algébrö̈des généralisent l'exemple 2.5 avec $\alpha$ rationel ; ce sont aussi les singularités des fonctions algébriques. Dans l'exemple 2.6, toutes les singularités de $g$ au dessus de $\mathbb{C}$ sont algébroïdes (et donc isolées) bien que leur projection soit dense. Dans l'exemple 2.7, toutes les singularités sont algébroïdes exceptée celle au dessus de 0 qui n'est même pas isolée. 


\section{Fonctions algébroïdes}

Définition 3.1. - On dit que $f$ définit une fonction algébrö̈de sur $\Omega$ si toute singularité de $f$ au dessus de $\Omega$ est algébrö̈de.

Exemple 3.2. - Si $\pi: \mathcal{S} \rightarrow \Omega$ est une application holomorphe propre (non nécessairement finie) d'une surface de Riemann $\mathcal{S}$ sur $\Omega$ et si $g: \mathcal{S} \rightarrow \mathbb{C}$ est une fonction méromorphe, alors la fonction multiforme $f$ définie sur $\Omega$ par $(\pi, g): \mathcal{S} \rightarrow \Omega \times \mathbb{C}$ est algébroïde sur $\Omega$. Les fonctions algébroïdes ainsi construites satisfont en outre à la propriété suivante. Elles ne possèdent qu'un nombre fini de déterminations dès que l'on restreint le prolongement analytique à un ouvert relativement compact dans $\Omega$. La fonction $f$ de l'exemple 2.6 est algébrö̈de sur $\mathbb{C}$ mais ne satisfait pas à cette dernière propriété.

Proposition 3.3. - Si $f$ définit une fonction algébrö̈de sur $\Omega$, alors :

1. l'ensemble $\widetilde{\Sigma}$ des singularités de $f$ au dessus de $\Omega$ est dénombrable,

2. pour tout chemin $\gamma:[0,1] \rightarrow \Omega$ issu de $x_{0}$, il existe un sous-ensemble fini $\Sigma(\gamma) \subset \Sigma \cap \gamma([0,1]) \subset \Omega$ et un $\varepsilon>0$ tels que $f$ se prolonge analytiquement le long de tout chemin $\varepsilon$-proche de $\gamma$ évitant $\Sigma(\gamma)$ : $\gamma^{\prime}:[0,1] \rightarrow \Omega, \gamma(0)=x_{0},\left|\gamma^{\prime}(t)-\gamma(t)\right|<\varepsilon$ et $\gamma^{\prime}(] 0,1[) \cap \Sigma(\gamma)=\emptyset$ conduisant éventuellement $f$ vers une singularité lorsque $\gamma^{\prime}(1)=$ $\gamma(1)$.

En particulier, l'ensemble des valeurs prises par les différentes déterminations $\left\{f_{\gamma^{\prime}}\left(x_{1}\right) ; \gamma^{\prime}\right.$ comme au dessus avec $\left.\gamma^{\prime}(1)=x_{1}\right\} \subset \overline{\mathbb{C}}$ est fini.

Remarque 3.4. - Dans la proposition précédente, l'ensemble $\Sigma(\gamma)$ joue le rôle des singularités intermédiaires possibles qu'il suffit de contourner (en perturbant $\gamma$ ) de façon à poursuivre le prolongement analytique de $f$ jusqu'à $x_{1}$.

Cette proposition est démontrée au début de [11]; la preuve que nous donnons ici a l'avantage de montrer qu'elle reste vraie, à la finitude près de $\Sigma(\gamma)$, sous l'hypothèse beaucoup plus faible que toute singularité de $f$ sur $\Omega$ est isolée. 
Lemme 3.5. - Si toute singularité de $f$ au dessus de $\Omega$ est isolée, alors l'ensemble $\widetilde{\Sigma}$ des singularités de $f$ au dessus de $\Omega$ est au plus dénombrable.

Démonstration. - C'est une adaptation du lemme de PoincaréVolterra. À toute singularité $f_{\gamma}$, on peut associer une suite de disques $D_{0}, D_{1}, \ldots, D_{n} \subset \Omega$ recouvrant $\gamma$ et des fonctions holomorphes $f_{k}: D_{k} \rightarrow \mathbb{C}$ pour $k=1, \ldots, n-1$ tels que $f_{0} \equiv f$ au voisinage de $x_{0}$, $f_{k} \equiv f_{k-1}$ sur $D_{k-1} \cap D_{k}$ et la fonction multiforme définie sur $D_{n}$ par $f_{n-1}$ possède exactement une singularité au dessus de $D_{n}$, à savoir $f_{\gamma}$. Quitte à diminuer la taille des disques, nous pouvons de plus supposer leur rayon et les coordonnées de leur centre dans $\mathbb{Q}$. Étant donnée une telle suite de disques $D_{k}$, les fonctions $f_{k}$ sont bien définies par unicité du prolongement analytique ; il en est de même de la singularité $f_{\gamma}$ au dessus de $x_{1}$ : on peut oublier le chemin. Puisque l'ensemble de telles suites de disques est dénombrable, l'ensemble des singularités l'est aussi.

Remarque 3.6. - Supposons que l'on rencontre une singularité de $f$ en $t_{1}<1$ le long d'un chemin $\gamma:[0,1] \rightarrow \Omega$ issu de $x_{0}=\gamma(0)$ (i.e. le chemin $\left.\gamma\right|_{\left[0, t_{1}\right]}$ conduit $f$ vers une singularité) ; si cette singularité est isolée, alors on peut la contourner dans le sens suivant. Pour $\varepsilon>0$ suffisamment petit, on peut modifier $\gamma$ le long de la composante connexe ]$t_{1}^{-}, t_{1}^{+}\left[\operatorname{de} \gamma^{-1}\left(\left\{\left|x-x_{1}\right|<\varepsilon\right\}\right)\right.$ contenant $t_{1}$ de sorte que le nouveau chemin $\gamma_{1}$ obtenu ainsi évite $x_{1}:=\gamma\left(t_{1}\right): \gamma(] t_{1}^{-}, t_{1}^{+}[) \subset\left\{\left|x-x_{1}\right|<\varepsilon\right\} \backslash\left\{x_{1}\right\}$. Le long de $\gamma_{1}$, le prolongement analytique de $f$ peut être poursuivi un peu plus loin, i.e. au moins jusqu'à $t_{1}^{+}$. Attention, la détermination de $f$ obtenue en $\gamma\left(t_{1}^{+}\right)$dépend de la perturbation $\gamma_{1}$ choisie ; cependant, parce que la singularité est isolée, elle ne dépend que de la classe d'homotopie de $\gamma_{1}$ parmi ceux donnés par la construction précédente. Pour une singularité non isolée, cette construction ne serait plus véritablement locale et perdrait de son sens. 


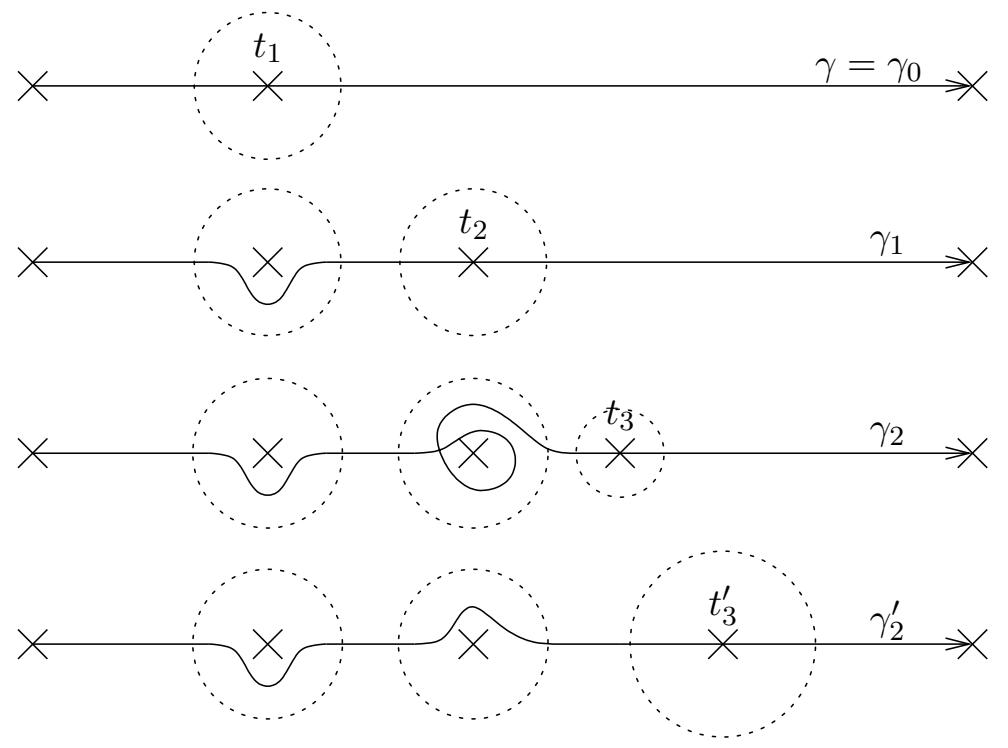

Figure 6. Contournement successif des singularités

Lemme 3.7. - Si toute singularité de $f$ au dessus de $\Omega$ est isolée, alors pour tout chemin $\gamma:[0,1] \rightarrow \Omega$ issu de $x_{0}=\gamma(0)$, quitte à répéter la construction de la remarque précédente un nombre fini de fois, on obtient un chemin $\gamma_{n}$ le long duquel $f$ admet un prolongement analytique (ou conduit vers une singularité).

En d'autres termes, on n'aura toujours qu'un nombre fini de singularités à contourner avant d'atteindre $\gamma(1)$, et ceci indépendamment de la façon dont on contourne ces "singularités intermédiaires".

Démonstration. - Supposons que pour certains choix successifs dans la façon de contourner les singularités intermédiaires, nous soyons amenés à effectuer une infinité de contournements successifs $\gamma_{0}=\gamma, \gamma_{1}, \gamma_{2}, \ldots$ mettant en évidence une infinité de singularités successives $0<t_{1}<t_{2}<$ $\cdots<1$, avec limite $0<t_{\infty} \leq 1$. Alors on peut choisir les modifications successives $\gamma_{n} \rightarrow \gamma_{n+1}$ de plus en plus petites de sorte que la suite $\gamma_{n}$ d'applications continues tende uniformément vers un chemin $\gamma_{\infty}$. Alors, $f$ possède une singularité en $\gamma\left(t_{\infty}\right)$ pour le prolongement analytique le long de $\gamma_{\infty}:\left[0, t_{\infty}\right] \rightarrow \Omega$ qui n'est, par construction, pas isolée. 
Remarque 3.8. - Les singularités successives $0<t_{1}<t_{2}<\ldots<$ $t_{n}<1$ rencontrées durant l'algorithme du Lemme précédent ainsi que leur nombre $n$ dépendent des choix successifs que l'on fait en les contournant. Par exemple, si la première singularité $t_{1}$ (qui, elle, ne dépend d'aucun choix) n'est pas à monodromie finie, alors il existe une infinité de façons de la contourner et la position de la singularité suivante $t_{2}$ peut dépendre entièrement de ce choix.

Lemme 3.9. - Si toute singularité de $f$ au dessus de $\Omega$ est isolée à monodromie finie, alors pour tout chemin $\gamma:[0,1] \rightarrow \Omega$ issu de $\gamma(0)=$ $x_{0}$, il existe un sous-ensemble fini $\Sigma(\gamma) \subset \Sigma \cap \gamma(] 0,1[) \subset \Omega$ tel que l'on ait la propriété (2) de la proposition 3.3.

Démonstration. - Supposons qu'il existe une infinité de singularités intermédiaires pour le prolongement algébroide le long de $\gamma$. Puisque la première singularité $\gamma\left(t_{1}\right)$ est à monodromie finie, il n'existe qu'un nombre fini de façons essentiellement distinctes de la contourner. Pour au moins un de ces choix $\gamma_{1}$, il doit rester une infinité de singularités intermédiaires possibles pour le prolongement analytique le long de $\gamma_{1}$. En répétant cette opération indéfiniment, on est amené à contourner une infinité de singularités le long de $\gamma$, ce qui contredit le Lemme précédent.

\section{Les théorèmes de Painlevé : des équations différentielles aux feuilletages}

Nous sommes maintenant en mesure d'énoncer le premier théorème de Painlevé :

Théorème (I). - Soient $P, Q \in \mathbb{C}[x, y]$ et considérons l'équation différentielle :

$$
\text { (E) } \quad \frac{d y}{d x}=\frac{P(x, y)}{Q(x, y)}
$$

Il existe un ensemble fini de points $\Sigma_{E} \subset \mathbb{C}$ sur lequel se projettent toutes les singularités non algébrö̈des au dessus de $\mathbb{C}$ de toute solution locale $y=f\left(x, x_{0}, y_{0}\right) d e(E)$. 
Ainsi, toute solution est une fonction algébroïde sur $\Omega=\mathbb{C} \backslash \Sigma_{E}$. En particulier, l'exemple 2.8 ne peut pas se produire : les solutions d'équations différentielles d'ordre 1 ne rencontrent pas de frontière naturelle au prolongement analytique.

Remarque 4.1. - La projection d'une singularité algébrö̈de de $f\left(x, x_{0}, y_{0}\right)$ dépend de la condition initiale $y_{0}$ et décrit tout $\Omega$ lorsque l'on décrit toutes les solutions. Aussi, on les appelle points critiques mobiles dans la littérature.

Introduisons maintenant les concepts géométriques qui se cachent derrière cet énoncé. L'idée de Paul Painlevé était de considérer l'équation différentielle $(E)$ comme un feuilletage singulier par courbes (à savoir les graphes des solutions) dans le portrait de phases $(x, y) \in \mathbb{C} \times \overline{\mathbb{C}}$ (Voir aussi $[\mathrm{Re}]$ ). Au voisinage de tout point $\left(x_{0}, y_{0}\right) \in \mathbb{C}^{2}$ satisfaisant $Q\left(x_{0}, y_{0}\right) \neq 0$, les graphes des solutions locales $y=f(x)$ dont les conditions initiales $f\left(x_{0}\right)$ sont proches de $y_{0}$ sont les courbes de niveaux d'une submersion $H(x, y)$ (Théorème de Cauchy à paramètre). Précisément, il existe un voisinage $U \ni\left(x_{0}, y_{0}\right)$ sur lequel $H: U \rightarrow \mathbb{C}$ est bien définie et régulière et telle que, pour toute condition initiale $\left(x_{1}, y_{1}\right) \in U$, la solution locale $f\left(x, x_{1}, y_{1}\right)$ est donnée par l'équation fonctionnelle implicite $H\left(x, f\left(x, x_{1}, y_{1}\right)\right)=H\left(x_{1}, y_{1}\right)$. Ainsi, les graphes de solutions locales définissent un feuilletage régulier par courbes dans un voisinage de $\left(x_{0}, y_{0}\right)$. Ce feuilletage $\mathcal{F}$ est globalement défini sur le complémentaire de $\{Q(x, y)=0\}$. Il s'étend, le long de $\{Q(x, y)=0\}$, au voisinage de chaque point $\left(x_{0}, y_{0}\right)$ satisfaisant $P\left(x_{0}, y_{0}\right) \neq 0$ par les mêmes arguments, après permutation du rôle des variables $x$ et $y$, i.e. simplement en considérant l'équation différentielle $\frac{d x}{d y}=\frac{Q(x, y)}{P(x, y)}$. Le feuilletage régulier $\mathcal{F}$ est ainsi bien défini sur le complémentaire $\mathbb{C}^{2} \backslash\{P(x, y)=Q(x, y)=0\}$. Quitte à diviser $P$ et $Q$ par un facteur commun, nous pouvons toujours supposer ces polynômes premiers entre eux. Ainsi, l'ensemble singulier $\{P(x, y)=Q(x, y)=0\}$ consiste en un nombre fini de points, à savoir les points d'indétermination du second membre de $(E)$. On dit que $\mathcal{F}$ est un feuilletage singulier à singularités isolées sur $\mathbb{C}^{2}$. 


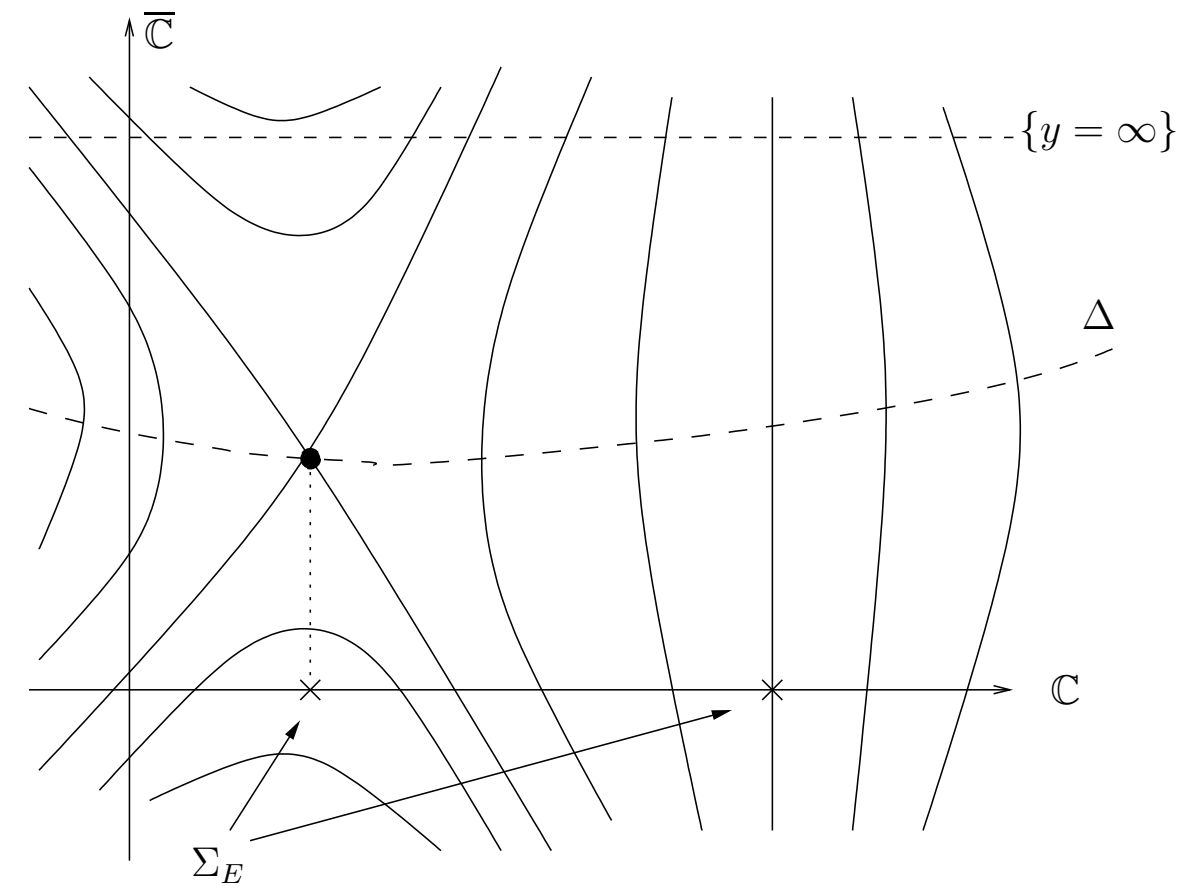

Figure 7 . Le feuilletage $\mathcal{F}$ et les singularités fixes $\Sigma_{E}$

Le feuilletage $\mathcal{F}$ s'étend en un feuilletage singulier à singularités isolées sur $\mathbb{C} \times \overline{\mathbb{C}}$ de la manière suivante. Supposons $P=P_{m}$ de degré $m \in \mathbb{N}$ et $Q=Q_{n}$ de degré $n \in \mathbb{N}$ en la variable $y$. Après changement de variable $Y=\frac{1}{y}$, il vient :

$$
\text { (E) } \quad \frac{d Y}{d x}=-Y^{2} \frac{P_{m}\left(x, \frac{1}{Y}\right)}{Q_{n}\left(x, \frac{1}{Y}\right)}=\frac{\widetilde{P}(x, Y)}{\widetilde{Q}(x, Y)}
$$

où $\widetilde{P}$ et $\widetilde{Q}$ sont les polynomes premiers entre eux donnés par :

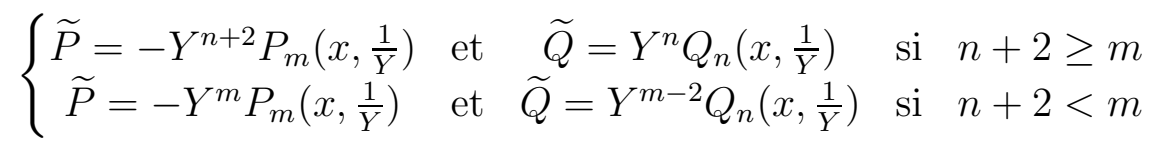

On développe de nouveau les arguments précédents pour étendre $\mathcal{F}$ en un feuilletage singulier à singularités isolées au voisinage de la droite à l'infini :

$$
L_{\infty}=\mathbb{C} \times \overline{\mathbb{C}} \backslash \mathbb{C}^{2}=\{y=\infty\}
$$


On note $\Sigma_{\mathcal{F}}$ l'ensemble (fini) des singularités de $\mathcal{F}$, c'est à dire des points de $\mathbb{C} \times \overline{\mathbb{C}}$ au voisinage desquels $\mathcal{F}$ ne s'étend pas comme feuilletage régulier :

$$
\begin{gathered}
\left.\Sigma_{\mathcal{F}}\right|_{\mathbb{C}^{2}}=\left\{\left(x_{0}, y_{0}\right) \in \mathbb{C}^{2} ; P\left(x_{0}, y_{0}\right)=Q\left(x_{0}, y_{0}\right)=0\right\}, \\
\left.\Sigma_{\mathcal{F}}\right|_{L_{\infty}}=\left\{\left(x_{0}, \infty\right) ; \widetilde{P}\left(x_{0}, 0\right)=\widetilde{Q}\left(x_{0}, 0\right)=0\right\} .
\end{gathered}
$$

On introduit aussi la courbe discriminante $\Delta$ comme l'ensemble des points de tangence entre le feuilletage $\mathcal{F}$ et la fibration verticale :

$$
\left.\Delta\right|_{\mathbb{C}^{2}}=\{(x, y) ; Q(x, y)=0\} \quad \text { et }\left.\quad \Delta\right|_{L_{\infty}}=\{(x, \infty) ; \widetilde{Q}(x, 0)=0\}
$$

Nous allons distinguer les différentes positions possibles du feuilletage $\mathcal{F}$ vis à vis de la fibration verticale en un point $\left(x_{0}, y_{0}\right) \in \mathbb{C} \times \overline{\mathbb{C}}$ suivant 5 catégories:

1. transversalité : $\left(x_{0}, y_{0}\right) \notin \Delta$,

2. tangence simple : $\mathcal{F}$ intersecte $\Delta$ transversalement en $\left(x_{0}, y_{0}\right)$,

3. tangence multiple : $\mathcal{F}$ intersecte $\Delta$ avec multiplicité en $\left(x_{0}, y_{0}\right)$,

4. singularité : $\left(x_{0}, y_{0}\right) \in \Sigma_{\mathcal{F}}$,

5. feuille verticale : $Q\left(x_{0}, y\right) \equiv 0$, i.e. $\left\{x=x_{0}\right\}$ est une feuille de $\mathcal{F}$.

L'ensemble $\Sigma_{E}$ est la projection sur $\mathbb{C}$ des points de type (4) et (5) :

$$
\Sigma_{E}=\left\{x_{0} ; Q\left(x_{0}, y\right) \equiv 0 \text { ou }\left(x_{0}, y_{0}\right) \in \Sigma_{\mathcal{F}} \text { pour un } y_{0} \in \overline{\mathbb{C}}\right\}
$$

Dans les exemples 2.5 et 2.7, $\Sigma_{E}$ est réduit à $\{0\}$. Dans l'exemple 2.6, on a $\Sigma_{E}=\left\{\zeta_{1}, \zeta_{2}, \zeta_{3}\right\}$ pour la première équation et toute solution $y=$ $f(x)$ est une fonction multiforme régulière de $x$ sur $\Omega:=\mathbb{C} \backslash\left\{\zeta_{1}, \zeta_{2}, \zeta_{3}\right\}$; par contre, $\Sigma_{E}$ est vide pour la seconde équation et toute solution $x=$ $g(y)$ est une fonction algébroïde transcendante de $x$ avec un ensemble dense de singularités dans le plan. 
Théorème (II). - Soient $P, Q \in \mathbb{C}[x, y]$ et considérons l'équation différentielle :

$$
\text { (E) } \quad \frac{d y}{d x}=\frac{P(x, y)}{Q(x, y)}
$$

Notons $\Omega$ le complémentaire dans $\mathbb{C}$ de l'ensemble $\Sigma_{E}$ donné par le Théorème I. Considérons une solution analytique locale $f\left(x, x_{0}, y_{0}\right)$ et un chemin $\gamma:[0,1] \rightarrow \Omega$ issu de $x_{0}=\gamma(0)$. Alors il existe une constante $\varepsilon>0$ telle que la flèche qui à une valeur initiale y proche de $y_{0}$ fait correspondre l'ensemble fini de valeurs :

$$
\varphi_{\gamma}(y):=\left\{f_{\gamma^{\prime}}\left(x_{1}, x_{0}, y\right) ; \begin{array}{l}
\gamma^{\prime}:[0,1] \rightarrow \Omega \text { est } \varepsilon \text {-proche de } \gamma \text { et } \\
\text { évite les singularités intermédiaires }
\end{array}\right\}
$$

définit, sur tout disque $D$ suffisamment petit centré en $y_{0}$, une fonction multivaluée de y donnée par un nombre fini de fonctions algébrö̈des (finies) sur D.

Si le concept de feuilletage n'est pas clairement défini dans $[\mathrm{Pa}]$, le Théorème II est bien connu des "feuilleteurs" dans le contexte suivant.

Remarque 4.2. - Le choix d'un prolongement algébroïde de $f\left(x, x_{0}, y_{0}\right)$ correspond au choix d'un chemin $\widetilde{\gamma}:[0,1] \rightarrow \Omega \times \overline{\mathbb{C}}$ relevant $\gamma$ dans la feuille $\mathcal{F}_{p_{0}}$ passant par le point $p_{0}=\left(x_{0}, y_{0}\right): \widetilde{\gamma}(0)=p_{0}$ et $\pi \circ \widetilde{\gamma}=\gamma$ (ici $\pi$ est la projection verticale). Il apparaitra clairement dans la suite que le prolongement obtenu est analytique (i.e. non singulier en $x_{1}$ ) dès que le feuilletage $\mathcal{F}$ est transverse à la verticale $T_{1}:=\left\{x=x_{1}\right\}$ au point d'arrivée $\widetilde{\gamma}(1)$. En d'autres termes, étant donné un chemin $\widetilde{\gamma}:[0,1] \rightarrow \Omega \times \overline{\mathbb{C}} \grave{a}$ valeurs dans une feuille joignant deux points $\gamma(i)$, $i=0,1$, en lesquels le feuilletage est transverse à la verticale $T_{i}$, il existe un $\varepsilon>0$ tel que la flèche $\widetilde{\gamma}^{\prime}(0) \mapsto \widetilde{\gamma}^{\prime}(1)$ qui fait se correspondre les extrémités de tous les chemins $\widetilde{\gamma}^{\prime}$ tangents au feuilletage, joignant $T_{0}$ à $T_{1}$ et $\varepsilon$-proches de $\widetilde{\gamma}$ définit une application uniforme et holomorphe sur un voisinage de $y_{0}$. C'est le lemme fondamental de l'holonomie.

Comme l'a maintes fois rappelé Painlevé (voir [Bo], p.142), il est important de comprendre que l'énoncé précédent n'est que local dans la variable $y$. Le prolongement analytique de la flèche $y \mapsto f\left(x_{1}, x_{0}, y\right)$ peut conduire à des singularités non algébroïdes dans le plan des y (voir $\S 6$ ). 


\section{Preuve du Théorème I}

La démonstration du premier théorème repose sur la compacité de la fibre verticale $\overline{\mathbb{C}}$. Nous commençons par prouver un cas particulier important.

Proposition 5.1. - Soient $P, Q \in \mathbb{C}[x, y]$ et considérons l'équation différentielle :

$$
\text { (E) } \quad \frac{d y}{d x}=\frac{P(x, y)}{Q(x, y)} .
$$

S'il existe un point $x_{0} \in \mathbb{C}$ au dessus duquel les solutions $y(x)$ de l'équation $(E)$ n'ont pas d'autre singularité que des pôles, alors $(E)$ est une équation de Riccati :

$$
\frac{d y}{d x}=\frac{a(x) y^{2}+b(x) y+c(x)}{Q(x)}
$$

où $a, b, c, Q \in \mathbb{C}[x]$ sont des polynômes d'une variable. De plus, on $a \Sigma_{E}=\{Q(x)=0\}$ et toute solution $y(x)$ est méromorphe sur le revêtement universel $\widetilde{\mathbb{C} \backslash \Sigma_{E}}$.

Démonstration. - L'absence de singularité algébroïde autre que des pôles au dessus de $x_{0}$ se traduit par la transversalité de la droite verticale $\left\{x=x_{0}\right\}$ avec le feuilletage $\mathcal{F}$ dans $\mathbb{C} \times \overline{\mathbb{C}}$. En particulier, $Q\left(x_{0}, y\right) \neq 0$ pour tout $y \in \mathbb{C}$, c'est à dire $Q=q_{0}(x)+q_{1}(x) y+\cdots+q_{n}(x) y^{n}$ avec $q_{0}\left(x_{0}\right) \neq 0$ et $q_{k}\left(x_{0}\right)=0$ pour $k=1, \ldots, n$. Après changement de coordonnées $Y:=1 / y$, il apparaît que $\widetilde{Q}\left(x_{0}, Y\right) \equiv q_{0}\left(x_{0}\right) Y^{k}$ où $k:=\sup \{m-2, n\}$. La transversalité de $\left\{x=x_{0}\right\}$ à $y_{0}=\infty$ se traduit par $\widetilde{Q}\left(x_{0}, 0\right) \neq 0$. Par suite, $n=0$ et $m \leq 2$ ce qui nous conduit à l'équation de Riccati. Dans ce cas, toute fibre verticale $\left\{x=x_{0}\right\}$, $Q\left(x_{0}\right) \neq 0$, est transverse au feuilletage. Par conséquent, la première projection $(x, y) \mapsto x$ induit sur chaque feuille $L$ un revêtement $L \rightarrow \mathbb{C} \backslash \Sigma_{E}$. Ainsi, $L$ est le graphe d'une fonction n'ayant que des pôles au dessus de $\mathbb{C} \backslash \Sigma_{E}$, à savoir les points d'intersection de $L$ avec la droite à l'infini $\{y=\infty\}$. 
Remarque 5.2. - Une équation différentielle dont les solutions sont toutes méromorphes est une équation de Riccati. En effet, les autres équations possèdent toujours des solutions avec singularités algébroïdes multiformes (points d'intersection du graphe avec $\Delta$ ). Cependant, très peu d'équations de Riccati ont des solutions méromorphes. L'exemple 2.6 :

$$
\frac{d y}{d x}=\frac{\alpha_{1}}{x-\zeta_{1}}+\frac{\alpha_{2}}{x-\zeta_{2}}+\frac{\alpha_{3}}{x-\zeta_{3}}
$$

est une équation de Riccati dont toute solution est multiforme.

La proposition 5.1 repose sur la trivialisation locale des points de type (1). Pour les points $\left(x_{0}, y_{0}\right)$ de type $(2)$, on peut encore donner des modèles locaux :

Lemme 5.3. - Soit $\mathcal{F}$ un feuilletage régulier défini au voisinage de l'origine $\underline{0} \in \mathbb{C}^{2}$ par une submersion $H: U \rightarrow \mathbb{C}$, avec une tangence verticale en $\underline{0}$. Supposons que le discriminant $\Delta:=\left\{\frac{\partial H}{\partial y}=0\right\}$ soit transverse à la verticale $\{x=0\}$ en $\underline{0}$. Alors, il existe un changement de coordonnées locales $\Phi(x, y)=(x, \phi(x, y))$ préservant la fibration verticale et envoyant $\mathcal{F}$ sur le feuilletage défini par $H_{k}(x, y)=x+y^{k}$, où $(k-1) \in \mathbb{N}^{*}$ est l'ordre de $\frac{\partial H}{\partial y}$ le long de $\Delta$. En d'autres termes, il existe un difféomorphisme local $\varphi$ tel que $H=\varphi \circ H_{k} \circ \Phi$.

Démonstration. - Par un changement de coordonnées préliminaire (préservant la fibration verticale), on peut déjà supposer $\Delta:\{y=0\}$. Alors, $\frac{\partial H}{\partial y}=y^{k-1} u(x, y)$ pour un entier $(k-1) \in \mathbb{N}^{*}$ et une unité holomorphe $u(x, y), u(0,0) \neq 0$. Par intégration, on obtient $H=f(x)+y^{k} \widetilde{u}(x, y)$ avec une nouvelle unité $\widetilde{u}(x, y)$ et une fonction $f(x)$ que l'on peut supposer s'annuler en 0, i.e. $H(0,0)=0$. Puisque $H$ est une submersion transverse à $\Delta, \varphi(x)=H(x, 0)$ est encore une submersion sur $\Delta$. Donc, $\varphi$ est un difféomorphisme local et $\widetilde{H}:=\varphi^{-1} \circ H$ est une nouvelle submersion définissant $\mathcal{F}$ de la forme $\widetilde{H}(x, y)=x+y^{k} \widetilde{u}(x, y)$. Finalement, si $v(x, y)$ désigne une racine primitive $k^{\text {ième }}$ de $\widetilde{u}(x, y)$, alors le changement de coordonnées défini par $\phi(x, y)=y \cdot v(x, y)$ satisfait $\widetilde{H}(x, y)=x+(\phi(x, y))^{k}=H_{k} \circ \Phi(x, y)$. 
Proposition 5.4. - Soit $T:=\left\{x=x_{0}\right\} \subset \mathbb{C} \times \overline{\mathbb{C}}$ une verticale transverse à $\Delta$ (tout point le long de $T$ est de type (1) ou (2)). Alors, on peut trouver un disque $D \subset \mathbb{C}$ centré en $x_{0}$ tel que la projection verticale restreinte au voisinage tubulaire $D \times \overline{\mathbb{C}}$ induise un revêtement ramifié des feuilles (après restriction) sur D. De plus, chacun de ces revêtements ramifiés a au plus un branchement. Les multiplicités de branchement possibles sont les multiplicités $k_{1}, \ldots, k_{n} \in \mathbb{N}^{*}$ de $Q(x, y)$ et $\widetilde{Q}(x, Y)$ le long des composantes irréductibles non verticales de $\Delta$. En d'autres termes, toute solution $f\left(x, x_{0}, y_{0}\right)$ dans $x \in D$ est ou bien uniforme, ou bien algébrö̈de avec exactement une singularité et $k_{i}$ déterminations pour un $i \in\{1, \ldots, n\}$.

Démonstration. - Elle se déduit par un argument de compacité appliqué aux modèles locaux donnés le long de la verticale par le Lemme 5.3.

Exemple 5.5. - Le feuilletage globalement donné par $H_{k}=x+y^{k}$ correspond à l'équation différentielle $\frac{d y}{d x}=-\frac{1}{k y^{k-1}}$. Toute solution $y=$ $\left(x_{0}+y_{0}^{k}-x\right)^{1 / k}$ possède exactement une singularité algébroïde en $x_{1}:=$ $x_{0}+y_{0}^{k}$. La position de cette singularité dépend des conditions initiales : $x_{1}$ est un point critique mobile.

Exemple 5.6. - Pour $n>2$, l'équation différentielle :

$$
\text { (E) } \frac{d y}{d x}=\frac{a_{n}(x) y^{n}+\cdots+a_{1}(x) y+a_{0}(x)}{Q(x)},
$$

où $a_{i}$ et $Q$ désignent des polynômes en $x$, a pour discriminant $\Delta=$ $L_{\infty} \cup\{Q(x)=0\}$ avec multiplicité $k=n-1$ le long de $L_{\infty}$.

Exemple 5.7. - Le feuilletage globalement défini par $H=\frac{x-y^{3}}{1-y}$ correspond à l'équation différentielle $\frac{d y}{d x}=\frac{y-1}{2 y^{3}-3 y^{2}+x}$. Son discriminant possède une tangente verticale à l'origine. La solution $f(x, 0,0)=x^{1 / 3}$ possède exactement une singularité algébroïde en 0 avec 3 déterminations. Puisque $y=f\left(x, x_{0}, y_{0}\right)$ est l'inverse de $x=g(y)=y^{3}-c y+c, c=$ $H\left(x_{0}, y_{0}\right)$, elle a 2 singularités algébrö̈des avec 2 déterminations locales autour de chacune d'elles ; d'un point de vue global, elle possède 3 déterminations. Lorsque $c \rightarrow 0$, ces deux singularités bifurquent en une seule d'ordre plus grand. 
Remarque 5.8. - Dès que l'on autorise la présence de singularités dans $\Delta$, on ne peut plus espérer obtenir de modèles polynomiaux. En effet, si l'on considère le cas le plus simple où $\Delta$ est à croisements ordinaires, disons $\Delta=\left\{y^{2}-x^{2}=0\right\}$, alors on peut mettre en évidence l'invariant suivant. Notons $\Delta_{1}=\{y-x=0\}$ et $\Delta_{2}=\{y+x=$ $0\}$ et définissons un germe de difféomorphisme $h: \Delta_{1} \rightarrow \Delta_{1}$ de la façon suivante. Envoyons d'abord $p \in \Delta_{1}$ sur un point $p^{\prime} \in \Delta_{2}$ par projection verticale, c'est à dire suivant le feuilletage vertical, puis envoyons $p^{\prime}$ sur l'unique point $h(p) \in \Delta_{1}$ qui est dans la même feuille pour $\mathcal{F}$. Si $H$ est une submersion définissant le feuilletage $\mathcal{F}$, alors $h(x)=h_{1}^{-1} \circ h_{2}$ où $h_{1}(x)=H(x, x)$ et $h_{2}(x)=H(x,-x)$. Après changement de coordonnées préservant le feuilletage vertical, c'est à dire de la forme $\Phi(x, y)=(\varphi(x), \phi(x, y)), h$ se trouve conjugué par $\varphi$. Par exemple, la submersion $H=x+\left(\frac{y^{3}}{3}-x^{2} y+\frac{2}{3} x^{3}\right) u(x), u(0)=0$, est de discriminant $\Delta=\left\{y^{2}-x^{2}=0\right\}$ et réalise le difféomorphisme $h(x)=x+\frac{4}{3} x^{3} u(x)$. Donc, tout difféomorphisme $h$ tangent à l'identité à l'ordre 2 est réalisable comme invariant local d'un tel feuilletage. La classification de tels difféomorphismes modulo conjugaison analytique donne naissance aux modules d'Écalle-Malgrange-Voronin qui sont fonctionnels, plus grand que l'espace des polynômes.

Lemme 5.9. - Soit $\mathcal{F}$ un feuilletage régulier défini au voisinage de $(0,0) \in \mathbb{C}^{2}$ par une submersion $H: U \rightarrow \mathbb{C}, H(0,0)=0$. Supposons que $\{H(x, y)=0\}$ ne soit pas la droite verticale $\{x=0\}: \frac{\partial H}{\partial y}(0, y)$ est d'ordre fini $k \in \mathbb{N}$ en $y=0$. Alors il existe un disque $D$ centré en $0 \in \mathbb{C}$ et $\varepsilon>0$ tels que, pour $|c| \leq \varepsilon$, les solutions $y=f(x)$ de l'équation implicite $H(x, y)=c$ sont algébroïdes sur $D$ avec $\leq k+1$ déterminations et au plus $k$ singularités. Plus précisément, pour $c=0$, il y a seulement une solution $y(x)$ avec seulement une singularité en $x=0$ et $k+1$ déterminations autour. Pour $c \neq 0$ suffisament proche de 0 , il y a encore une unique solution $y(x)$ avec $\leq k$ singularités $\neq 0$ et $k+1$ déterminations autour. Alors que $|c|$ croît, les singularités s'échappent par le bord de $D$ faisant croître le nombre de solutions distinctes. Finalement, pour $|c|=\varepsilon$, il y a exactement $k+1$ solutions distinctes uniformes. 
Démonstration. - Lorsque $k=0$, le théorème des fonctions implicites donne des solutions uniformes $\varphi_{c}(x)$ analytiques sur un polydisque $(x, c) \in D \times D_{\varepsilon}$ centré en $(0,0)$. À partir de maintenant, supposons $k \geq 1$. Le théorème des fonctions implicites donne des solutions uniformes $x=\psi_{c}(y)$ à l'équation fonctionnelle $H\left(\psi_{c}(y), y\right)=c$ analytiques sur un polydisque $(y, c) \in D^{\prime} \times D_{\varepsilon}$ centré en $(0,0)$. Pour $c=0, \psi_{0}(y)$ possède un point critique s'annulant à l'ordre $k+1$ en $y=0$. Quitte à diminuer $D^{\prime}, \psi_{0}(y)$ est conjugué, sur un voisinage de l'adhérence $\overline{D^{\prime}}$, à la fonction $y \mapsto y^{k+1}$. En particulier, il existe 2 disques $D^{-} \subset D^{+}$centrés en $x=0$ tels que la frontière $\partial D^{\prime}$ soit envoyée par $\psi_{0}$ dans l'anneau $D^{+} \backslash \overline{D^{-}}$avec indice $k+1$. Quitte à diminuer $\varepsilon, \psi_{c}$ envoit encore $\partial D^{\prime}$ dans $D^{+} \backslash \overline{D^{-}}$avec indice $k+1$ et, par le théorème de Rouché, possède encore $k$ points critiques (comptés avec multiplicité) dans $D^{\prime}$ pour $|c| \leq \varepsilon$. Ceci signifie que pour pour tout sousdisque $D \subset D^{-}, \psi_{c}$ induit un revêtement ramifié $\psi_{c}^{-1}(D) \rightarrow D$ de degré $k+1$ avec au plus $k$ points critiques. Les solutions algébrö̈des $y(x)$ de l'énoncé sont les applications inverses de $\psi_{c}$, allant de $D$ vers les composantes connexes de $\psi_{c}^{-1}(D)$ ce qui prouve le premier point.

Il reste à montrer que les images des points critiques de $\psi_{c}$, qui deviennent les points singuliers des solutions $y(x)$, s'échappent de 0 et, par suite, de tout disque $D$ suffisamment petit lorsque $|c|$ croît. En fait, nous allons montrer que les singularités des solutions $y(x)$ sont des germes de fonctions algébroïdes non constantes de $c$ en 0 . Remarquons d'abord que les points critiques $y_{1}(c), \ldots, y_{k}(c)$ de $\psi_{c}$ sont les déterminations de fonctions algébroïdes de $c$ en $c=0$.

En effet, notons $\Delta$ la courbe discriminante de $\mathcal{F}, \Delta=\left\{\frac{\partial H}{\partial y}=0\right\}$. Dans les nouvelles coordonnées $(c, y):=(H(x, y), y)$, la fonction $H$ devient la première coordonnée et l'ancienne coordonnée $x=x(c, y)$ devient une submersion de discriminant $\Delta$. Alors, les points critiques $y_{1}(c), \ldots, y_{k}(c)$ sont les points d'intersection de la droite verticale $\{H=c\}$ avec la courbe $\Delta$. Donc, les points critiques sont les paramétrisations de Puiseux de $\Delta$ dans ces coordonnées et sont par suite algébroïdes au voisinage de 0 . Finalement, les points singuliers $\zeta_{i}(c)$ des solutions $y(x)$ sont donnés par $\zeta_{i}(c)=\psi_{c}\left(y_{i}(c)\right)$ et sont aussi algébroïdes (composition de d'une série de Puiseux par une série entière). 
Puisque $H$ possède une tangente isolée avec la droite verticale $\{x=0\}$ en $(0,0)$, le graphe $\{H(x, y)=c\}$ de $\psi_{c}$ intersecte transversalement la droite $\{x=0\}$ pour $c \neq 0$ et tout fonction inverse correspondante $y(x)$ est analytique en 0 . Donc, aucun de ces germes algébroïdes $\zeta_{i}(c)$ ne peut être constant $\equiv 0$. En particulier, pour $|c|=\varepsilon$, chacune de ces déterminations s'est échappé de tout disque suffisamment petit $D$.

Remarque 5.10. - Dans l'exemple 5.7, les points critiques des solutions $x(y)$ sont les 2 déterminations de la fonction algébrö̈de $\frac{c^{1 / 2}}{\sqrt{3}}=$ $\left\{y_{1}(c), y_{2}(c)\right\}$. Les points singuliers de l'inverse $y(x)$ sont les valeurs critiques de la fonction précédente, c'est à dire sont les 2 déterminations de la fonction algébroïde $c-\frac{2}{3 \sqrt{3}} c^{3 / 2}=\left\{\zeta_{1}(c), \zeta_{2}(c)\right\}$. Dans la remarque 5.8, les points singuliers sont 2 fonctions uniformes données par $\zeta_{1}(c)=h_{1}^{-1}(x)$ et $\zeta_{2}(c)=h_{2}^{-1}(x)$. L'invariant $h$ discuté dans la remarque 5.8 n'est autre que l'application permuttant les singularités. Aussi, dans l'exemple 5.7, si $\Delta$ est paramétré par $z \mapsto\left(3 z^{2}-2 z^{3}, z\right)$, alors $\left.H\right|_{\Delta}(z)=z^{2}$. Donc, l'invariant $h$, vu comme application $\Delta \rightarrow \Delta$ est simplement $z \mapsto-z$ alors qu'il devient algébrö̈de lorsqu'il est vu dans la variable $x$.

Nous déduisons finalement du Lemme 5.9 la version quantitative suivante du Théorème I.

Corollaire 5.11. - Étant donnés l'équation différentielle:

$$
\text { (E) } \quad \frac{d y}{d x}=\frac{P(x, y)}{Q(x, y)}
$$

et $\Sigma_{E} \in \mathbb{C}$ définis comme précédemment, il existe un $k \in \mathbb{N}$ tel que toute solution est algébroïde sur $\Omega=\mathbb{C} \backslash \Sigma_{E}$ avec singularités ramifées $\grave{a}$ l'ordre $\leq k$. De plus, pour tout chemin différentiable $\gamma:[0,1] \rightarrow$ $\Omega$ et toute solution analytique locale $f\left(x, x_{0}, y_{0}\right), x_{0}=\gamma(0)$, le nombre de déterminations obtenues en $x_{1}=\gamma(1)$ par prolongement analytique le long de $\gamma$ contournant les singularités intermédiaires est borné par $(k+1)^{|\gamma| / \varepsilon(\gamma)}$ où $|\gamma|$ désigne la longueur de $\gamma$ et $\varepsilon(\gamma)>0$ est une fonction décroissante de la distance $d\left(\gamma([0,1]), \Sigma_{E}\right)$ de $\gamma$ à l'ensemble singulier $\Sigma_{E}$ (pour la distance sphérique sur $\overline{\mathbb{C}} \supset \mathbb{C}$ ). 
Démonstration. - Près de tout point $\left(x_{0}, y_{0}\right) \in \mathbb{C}^{2}$ satisfaisant $x_{0} \notin \Sigma_{E}$, le lemme 7 fournit une description des solutions de $(E)$ sur un voisinage ouvert de la forme $\left\{|x|<\varepsilon_{1},|H|<\varepsilon_{2}\right\}, \varepsilon_{1}, \varepsilon_{2}>0$. Par un argument de compacité, la fibre $\left\{x=x_{0}\right\}$ peut être recouverte par un nombre fini de tels voisinages. Il existe donc un disque $D\left(x_{0}\right)$ centré en $x_{0}$ tel que toute solution $y(x)$ de $(E)$ dans $D$ est algébroïde avec au plus $k$ points singuliers et au plus $k+1$ déterminations. L'entier $k$ est la multiplicité totale d'intersection entre $\Delta$ et la droite verticale $\left\{x=x_{0}\right\}$ et est alors indépendant de $x_{0}$. Tout sous-ensemble compact $K \subset \Omega$ (par exemple $\left.K=K_{r}:=\left\{x ; d\left(x, \Sigma_{E}\right) \geq r\right\}, r>0\right)$ est recouvert par un nombre fini de tels disques $D_{1}, \ldots, D_{N}$. Alors pour tout chemin $\gamma:[0,1] \rightarrow K$ et pour toute solution analytique locale $f\left(x, x_{0}, y_{0}\right), x_{0}=\gamma(0)$, on peut effectuer le prolongement algébroïde le long de $\gamma$ en recollant ensemble un nombre fini de solutions algébrö̈des dans les disques $D_{i}$ rencontré. Le nombre maximal de disques successifs $D\left(x_{i}\right)$ nécessaire pour recouvrir $\gamma$ est borné par la longueur $|\gamma|$. En effet, fixons un $\varepsilon>0$ tel que les disques $\varepsilon$-rognés $D_{i}^{-}:=\left\{x \in D_{i} ; d\left(x, \partial D_{i}\right)>\varepsilon\right\}$ recouvrent encore $K$. Alors si $\gamma\left(t_{0}\right)$ est dans un $D_{i}^{-}, \gamma(t)$ reste dans $D_{i}$ pour $t \in\left[t_{0}, t_{0}+\varepsilon\right]$ avant de changer pour un autre disque $D_{j}^{-}$. Ainsi, on a besoin de changer au plus $\frac{|\gamma|}{\varepsilon}$ fois de disques $D_{i}$ pour pouvoir effectuer le prolongement algébroïde le long de $\gamma$. En d'autres termes, au plus $\frac{|\gamma|}{\varepsilon}+1$ disques distincts ont été rencontrés durant le prolongement analytique et au plus $(k+1)^{\left(\frac{|\gamma|}{\varepsilon}+1\right)}$ déterminations distinctes peuvent être obtenues pour $f\left(x_{1}, x_{0}, y_{0}\right), x_{1}=\gamma(1)$. 


\section{Preuve du théorème II et l'exemple de Painlevé}

Preuve du théorème II. - Soient $f\left(x, x_{0}, y_{0}\right)$ et $\gamma:[0,1] \rightarrow \Omega$ comme dans l'énoncé et soit $\Sigma_{\gamma}(f)$ l'ensemble des singularités intermédiaires possibles (voir propriété $(\mathrm{B}) \mathrm{du} \S 3$ ). Tout prolongement algébroïde $f_{\gamma}$ va donner naissance à une composante irréductible de $\varphi$. Un tel prolongement est caractérisé, d'après la remarque 14, par un relèvement :

$$
\widetilde{\gamma}:[0,1] \rightarrow L_{0} \subset \mathbb{C} \times \overline{\mathbb{C}}, \Pi_{1} \circ \widetilde{\gamma}=\gamma \quad \text { avec } \quad \widetilde{\gamma}(0)=\left(x_{0}, y_{0}\right)
$$

dans la feuille $L_{0}$ passant par $\left(x_{0}, y_{0}\right)$. Soient $\widetilde{\gamma}_{1}, \ldots, \widetilde{\gamma}_{N}:[0,1] \rightarrow \Omega \times \overline{\mathbb{C}}$ tous les relèvements possibles de $\gamma$, caractérisant tous les prolongements algébrö̈des de $f$. En particulier, tous ces chemins coïncident pour $t$ petit et bifurquent au fûr et à mesure que l'on rencontre les points de $\Sigma_{\gamma}(f)$.

D'après le lemme 7 et par compacité de la fibre, pour tout $t \in[0,1]$, on peut trouver un disque $D$ centré en $\gamma(t)$ tel que toute feuille $L$ du feuilletage $\left.\mathcal{F}\right|_{D}$ restreinte au cylindre $D \times \overline{\mathbb{C}}$ est un revêtement ramifié sur $D$. Notons $L_{l}$ la feuille locale contenant $\widetilde{\gamma}_{l}(t)$. Quitte à diminuer $D$, on peut supposer que chaque application $\pi: L_{l} \rightarrow D$ est ou bien un difféomorphisme, ou bien un revêtement ramifié ne ramifiant qu'au dessus de $\widetilde{\gamma}_{l}(t)$. Dans ce dernier cas, remarquons que $\gamma(t) \in \Sigma_{\gamma}(f)$ et, si l'ordre de branchement est $q$, alors $q$ relèvements $\widetilde{\gamma}_{l}$ coincident jusqu'à $t$ et deviennent distincts juste après. Par compacité du chemin $\gamma$, on peut extraire de ces disques un recouvrement fini, disons :

$$
D_{k} \supset \gamma\left(\left[t_{k}, t_{k+1}\right]\right) \quad \text { où } \quad 0=t_{0}<t_{1}<\cdots<t_{n}<t_{n+1}=1 \text {. }
$$

Pour $k=0, \ldots, n$ et $l=1, \ldots, N$, notons $L_{k, l}$ la feuille du feuilletage restreint $\left.\mathcal{F}\right|_{D_{k}}$ contenant $\widetilde{\gamma}_{l}\left(t_{k}\right)$. En particulier, $L_{k, l} \cap L_{k+1, l}$ est envoyé difféomorphiquement sur $D_{k} \cap D_{k+1}$ par $\pi$. Soit $H_{k, l}: U_{k, l} \rightarrow \mathbb{C}$ une submersion definissant le feuilletage sur un voisinage tubulaire $U_{k, l}$ de $L_{k, l}$. Après composition (à gauche) des $H_{k, l}$ par des difféomorphismes convenables, on peut supposer $H_{0, l}\left(x_{0}, y\right)=y$ et $H_{k+1, l} \equiv H_{k, l}$ au voisinage de $\widetilde{\gamma}_{l}\left(t_{k+1}\right)$. Pour $\varepsilon>0$ suffisamment petit, on peut supposer que, pour tout $\left|y_{0}^{\prime}-y_{0}\right|<\varepsilon$, l'ensemble $\left\{x \in D_{k}\right.$ et $\left.H_{k, l}(x, y)=y_{0}^{\prime}\right\}$ n'est pas vide et est une feuille complète pour le feuilletage restreint $\left.\mathcal{F}\right|_{D_{k}}$. Pour simplifier, remplaçons $U_{k, l}$ par l'ouvert $\left\{x \in D_{k}\right.$ et $\left.\left|H_{k, l}(x, y)-y_{0}\right|<\varepsilon\right\}$. 
Soit $\gamma^{\prime}:[0,1] \rightarrow \Omega$ une $\varepsilon$-perturbation de $\gamma$ avec $\gamma^{\prime}(0)=x_{0}$. Si $\varepsilon>0$ est suffisamment petit, Alors $D_{k}$ reste un recouvrement ordonné pour $\gamma^{\prime}$. De plus, on peut supposer $\gamma^{\prime}\left(t_{k}\right) \in D_{k} \cap D_{k+1}$. Soit $\widetilde{\gamma}^{\prime}:[0,1] \rightarrow \mathbb{C} \times \overline{\mathbb{C}}$ un relèvement de $\gamma^{\prime}$ dans le feuilletage correspondant à un prolongement algébroïde d'une solution $f\left(x, x_{0}, y_{0}^{\prime}\right)$ avec $\left|y_{0}^{\prime}-y_{0}\right|<\varepsilon$. Il s'en suit que $\widetilde{\gamma}^{\prime}(1)=\left(x_{1}, y_{1}^{\prime}\right)$ où $x_{1}=\gamma(1)$ et $y_{1}^{\prime}$ est l'une des valeurs prises par $\varphi_{\gamma}$ en $y_{0}^{\prime}$. De plus, toute valeur de $\varphi_{\gamma}$ est obtenue de cette manière.

À présent, nous sommes à même de prouver que $\widetilde{\gamma}^{\prime}$ reste proche de $\widetilde{\gamma}$. En effet, supposons que $\widetilde{\gamma}^{\prime}\left(t_{k}\right)$ soit contenu dans un $U_{k, l}$. Alors $\widetilde{\gamma}^{\prime}\left(t_{k}\right)$ est dans la feuille locale $L_{k}:=\left\{x \in D_{k}\right.$ et $\left.H_{k, l}(x, y)=y_{0}^{\prime}\right\}$. Puisque $\gamma^{\prime}\left(\left[t_{k}, t_{k+1}\right]\right)$ est contenu dans $D_{k}, \widetilde{\gamma}^{\prime}\left(\left[t_{k}, t_{k+1}\right]\right)$ est complètement contenu dans $L_{k}$. En particulier, quitte à remplacer l'indice $l$ par un autre convenable parmis ceux pour lesquels $H_{k, l}: U_{k, l} \rightarrow \mathbb{C}$ reste inchangé, $\widetilde{\gamma}^{\prime}\left(t_{k+1}\right)$ est dans $U_{k, l} \cap U_{k+1, l}$. Par récurrence, puisque $\widetilde{\gamma}^{\prime}(0) \in U_{0,1}$, il s'en suit qu'il existe un $l=1, \ldots, N$ tel que $\widetilde{\gamma}^{\prime}\left(t_{k}\right) \in U_{k, l}$ pour tout $k=0, \ldots, n$. Finalement, $\widetilde{\gamma}^{\prime}(1)=\left(x_{1}, y_{1}^{\prime}\right) \in L_{n}=\left\{x \in D_{n}\right.$ et $\left.H_{k, l}(x, y)=y_{0}^{\prime}\right\}$. Par conséquent, $y_{1}^{\prime}$ est la valeur prise en $y_{0}^{\prime}$ par une des déterminations des fonctions algébroïdes $\varphi_{l}(y)$ définies pour $\left|y-y_{0}\right|<\varepsilon$ par $H_{n, l}\left(x_{1}, \varphi_{l}(y)\right)=$ $y$.

L'énoncé original du théorème II parait ambigü aujourd'hui. Dans les Leçons de Stokholm, il est écrit (voir [Pa], tome 1, p.210) :

Soit $y_{0}$ la valeur de $y(x)$ pour $x=x_{0}$, et soit $y=\varphi\left(x, y_{0}, x_{0}\right)$ l'intégrale générale de (A). Si $\bar{x}, \overline{x_{0}}$ désignent deux valeurs numériques quelconques distinctes des valeurs $\xi$, la fonction $y=\varphi\left(\bar{x}, y_{0}, \overline{x_{0}}\right)$ ne présente dans tout le plan des $y_{0}$ (à distance finie ou infinie) que des points singuliers algébriques.

On aurait tendance à penser, en lisant ce texte, que pour tous points $x_{0}$ et $x_{1}$ distincts de $\Sigma_{E}$, tout chemin $\gamma$ les joignant et toute valeur $y_{1}=$ $f\left(x_{1}, x_{0}, y_{0}\right)$ obtenue en choisissant un relevé de $\gamma$ dans le feuilletage, le germe de fonction $\varphi:\left(\mathbb{C}, y_{0}\right) \rightarrow\left(\mathbb{C}, y_{1}\right)$ correspondant défini par la flèche $y \mapsto f\left(x_{1}, x_{0}, y\right)$ admet un prolongement algébroïde sur $\overline{\mathbb{C}}$. Cependant, la simple lecture de la preuve originale de Painlevé nous montre qu'il n'en est rien : le théorème prouvé est purement local. De plus, il est très facile de se convaincre que le germe $\varphi$ possède en général des singularités non algébroïdes. 
Exemple 6.1. - Si $P$ et $Q$ sont deux polynômes de degré $d \in \mathbb{N}^{*}$ suffisamment génériques, alors le feuilletage induit par l'équation différentielle correspondante :

$$
\text { (E) } \quad \frac{d y}{d x}=\frac{P(x, y)}{Q(x, y)}
$$

possède exactement $d^{2}$ points singuliers dans $\mathbb{C}^{2}$, tous hyperboliques. D'après le théorème de Poincaré, il existe, au voisinage d'un tel point $p \in$ $\mathbb{C}^{2}$, un système de cordonnées analytiques locales redressant le feuilletage sur celui défini par :

$$
\frac{d y}{d x}=\alpha \frac{y}{x} \quad \text { c'est à dire } \quad\left\{y \cdot x^{-\alpha}=\text { constante }\right\}
$$

pour un nombre complexe $\alpha \notin \mathbb{R}$. En particulier, le feuilletage possède deux courbes locales invariantes, lisses et transverses, au point $p$ qui n'ont aucune raison, ni l'une ni l'autre, d'être verticales. Ces deux feuille vont imanquablement intersecter n'importe quelle droite verticale $T=\left\{x=x_{0}\right\}$. Notons $L_{0}$ et $L_{1}$ ces deux feuilles, choisissons (le cas échéant) un point d'intersection respectif $p_{0}=\left(x_{0}, y_{0}\right) \in L_{0} \cap T$ et $p_{1}=\left(x_{0}, y_{1}\right) \in L_{1} \cap T$ ainsi qu'un chemin $\widetilde{\gamma}_{i}$ joignant $p_{i}$ à $p$ dans $L_{i}$ pour $i=1,2$. La concaténation $\widetilde{\gamma}:=\widetilde{\gamma}_{0} \cdot \widetilde{\gamma}_{1}^{-1}$ est un chemin allant de $p_{0}$ à $p_{1}$, tangent au feuilletage et changeant de feuille au point $p$. Au vu du modèle local, le germe d'application retour $\varphi:\left(\mathbb{C}, y_{0}\right) \rightarrow\left(\mathbb{C}, y_{1}\right)$ correspondant (défini comme dans l'énoncé du théorème II par les perturbations de $\widetilde{\gamma}$ tangentes au feuilletage et à extrémités dans $T$ ) est localement conjugué à l'application $y \mapsto\left(y-y_{0}\right)^{-\alpha}+y_{1}$ qui n'est pas algébroïde en $y_{0}$. C'est l'application de Dulac complexe de la singularité p. Bien sûr, le théorème II n'est pas contredit puisque l'on a choisi un chemin $\widetilde{\gamma}$ passant par une singularité du feuilletage et donc relevant un chemin $\gamma$ dans la base qui intersecte $\Sigma_{E}$. Cependant, toute détermination locale de $\varphi$ en un point $y_{0}^{\prime}$ proche de $y_{0}$ est un germe d'application donné par le théorème II qui, par construction, n'admet pas de prolongement algébroïde sur $\mathbb{C}$. 


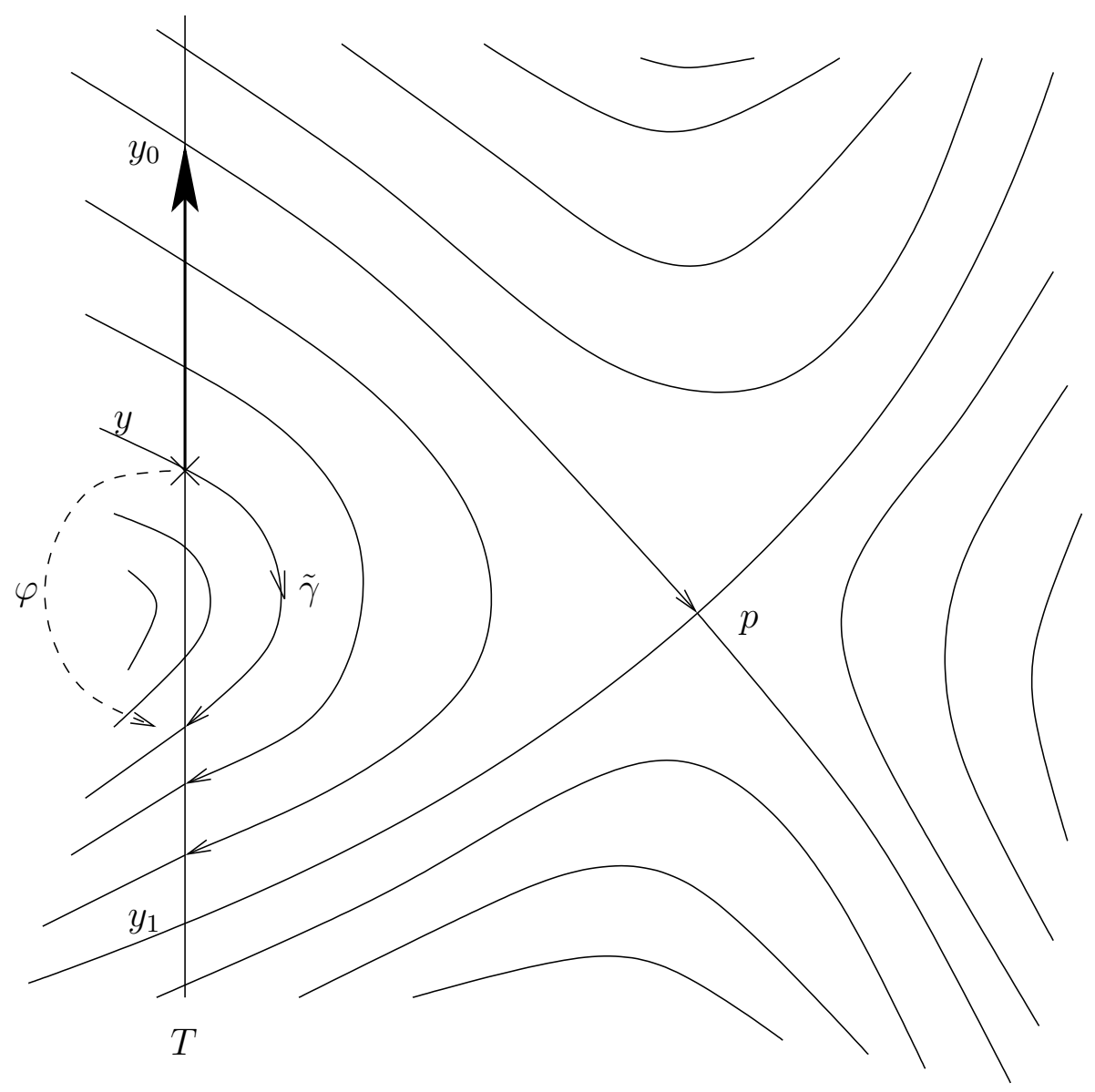

Figure 8. Exemple 6.1

L'énoncé original du théorème II a dû suscitter maintes critiques puisque Paul Painlevé a cru bon de rappeler, dans l'appendice du livre de Boutroux (voir [2], p.142-144) que l'énoncé n'est que local. Pour bien montrer qu'il avait en tête qu'une interprétation globale de son énoncé ne pouvait pas être vraie, il a même cru bon de donner le contre-exemple suivant. Comme nous l'avons décortiqué en détails, il est plus compliqué que le précédent mais très instructif pour d'autres raisons. Je remercie K. Okamoto de m'avoir signalé cet exemple en Février 2001. 
Exemple 6.2 ([2], p.142-144). — Considérons l'équation différentielle :

$$
\text { (E) } \quad \frac{d y}{d x}=\frac{y}{x(y+1)} .
$$

Son intégration nous conduit à l'intégrale première globale $H(x, y)=$ $\frac{y e^{y}}{x}$ et les solutions globales sont définies par l'équation implicite $H\left(x, f\left(x, x_{0}, y_{0}\right)\right)=H\left(x_{0}, y_{0}\right)$. Après compactification dans $\overline{\mathbb{C}} \times \overline{\mathbb{C}}$, le feuilletage $\mathcal{F}$ possède exactement 4 droites invariantes, à savoir $\{x=0\}$, $\{x=\infty\},\{y=0\}$ et $\{y=\infty\}$. Toute feuille autre que ces droites est transcendante et admet une paramétrisation uniforme globale par $y \in \mathbb{C}$, à savoir $y \mapsto c \cdot y e^{y}$. Néanmoins, les solutions correspondantes $y=f\left(x, x_{0}, y_{0}\right)$ sont des fonctions multiformes de $x$ comme nous allons le voir. Par ailleurs, $\mathcal{F}$ possède exactement 4 points singuliers situés aux intersections de ces droites invariantes.

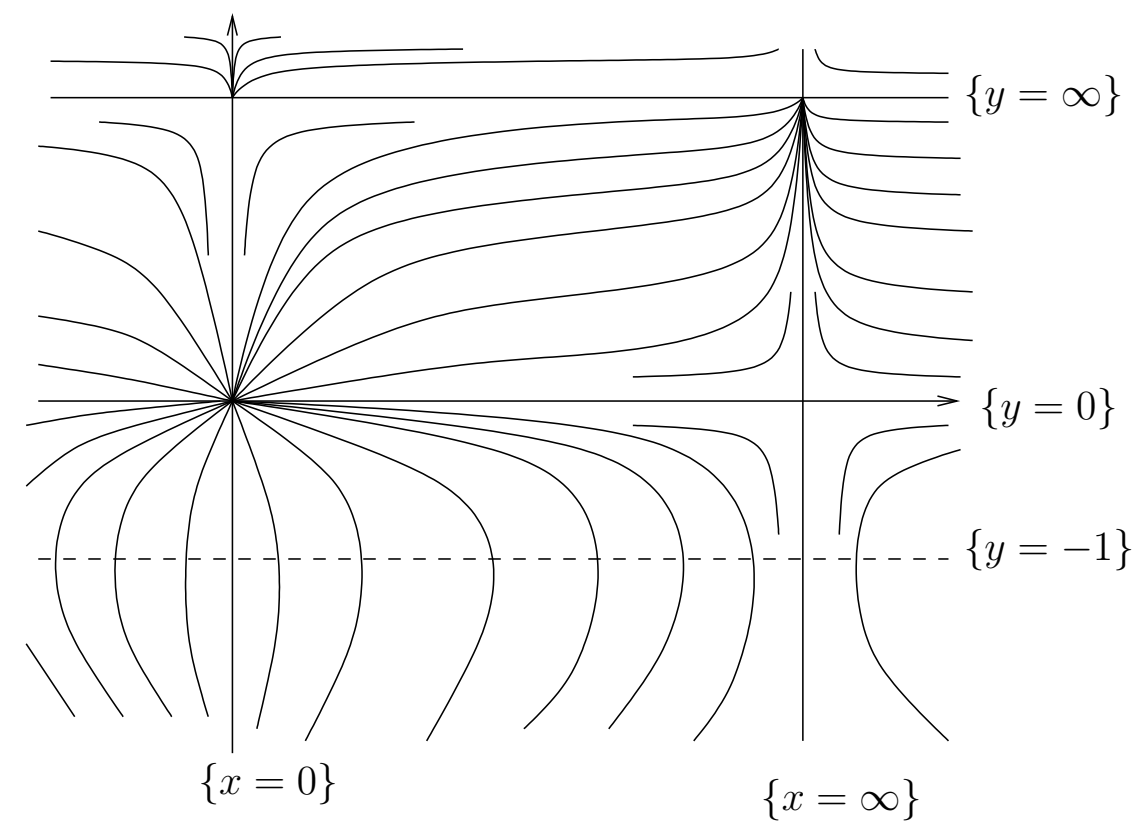

Figure 9. Feuilletage associé à l'exemple 6.2

Précisément, $(0,0)$ est un noud $x(1+y) d y-y d x=0$ et est localement conjugué à $x d y=y d x$ d'après le théorème de Poincaré. Ceci signifie qu'il existe un changement de coordonnées analytiques locales envoyant 
les feuilles de $\mathcal{F}$ sur celles du feuilletage radial $\left\{\frac{y}{x}=\right.$ constant $\}$. De même, $(\infty, 0)$ est une singularité selle $X(1+y) d y+y d X=0$ (ici $X=\frac{1}{x}$ ) et est localement conjuguée à sa partie linéaire $X d y+y d X=0$. Finalement, les singularités en $(0, \infty)$ et en $(\infty, \infty)$ sont des selle-nœuds respectivement donnés par $x(1+Y) d Y)+Y^{2} d x$ (où $Y=\frac{1}{y}$ ) et $\left.X(1+Y) d Y\right)-Y^{2} d X$. Donc $\Sigma_{E}=\{0, \infty\}$. Enfin, le lieu des tangences verticales de $\mathcal{F}$, en dehors des 2 droites verticales invariantes, est donné par $\Delta=\{y=-1\}$. La feuille $L_{x_{0}, y_{0}}$ correspondant à n'importe quelle solution (non constante) $y=f\left(x, x_{0}, y_{0}\right)$ intersecte $\{y=0\}$ une fois en $(0,0), \Delta$ une fois en $\left(x_{1},-1\right)$ avec $x_{1}=-\frac{x_{0}}{y_{0} e^{y_{0}+1}}$ et accumule la droite horizontale $\{y=\infty\}$. Ceci se déduit aisément de la parametrisation par la variable $y$. Donc $f\left(x, x_{0}, y_{0}\right)$ possède exactement 3 singularités, à savoir en $0, x_{1}$ et $\infty$, et possède une infinité de déterminations en tout autre point. Autour de $x=0$, une des branches possède une extension analytique et coïncide avec une des feuilles locales de la singularité radiale de $\mathcal{F}$. Les autres branches tendent vers $\infty$ et se permutent transitivement lorsque l'on tourne autour de $x=0$. Autour de $x=\infty$, toutes les branches tendent vers $\infty$ et se permutent transitivement. Autour de $x_{1}$, seules 2 branches se permutent autour de la singularité algébroïde alors que toute autre branche possède une extension analytique en $x_{1}$. Ceci nous donne une description qualitative complète des solutions.

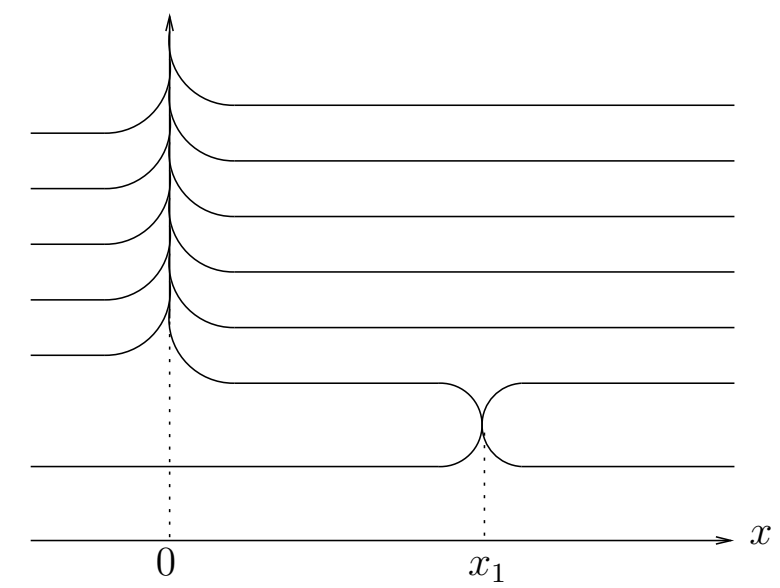

Figure 10. Graphe d'une solution de l'exemple 6.2 
Remarque 6.3. - Painlevé a soulevé le paradoxe suivant. Choisissons une solution analytique locale $f\left(x, x_{0}, y_{0}\right) \not \equiv 0$ en un point de $\Omega$, disons $x_{0}=1$ pour simplifier. En particulier, l'unique singularité algébroïde $x_{1}$ est supposée distincte de $x_{0}$, c'est à dire $y_{0} \neq-1$. Alors, toute détermination de $f(x)$ en $x_{0}$ peut être atteinte par prolongement analytique le long d'un lacet $\gamma$ d'indice 1 autour de $x_{1}$ mais d'indice 0 autour de 0. En d'autres termes, une infinité de branches sont permutées uniquement en tournant autour de la singularité algébroïde sans tourner autour des autres! En effet, choisissons un chemin $\sigma:[0,1] \rightarrow \Omega^{*}$, $\Omega^{*}:=\Omega \backslash\left\{x_{1}\right\}$, allant de $x_{0}$ vers un point $x_{0}^{\prime}$ proche de 0 le long duquel le prolongement analytique de $f(x)$ conduit à l'unique branche analytique en 0 . Alors, notons $\tau:[0,1] \rightarrow \Omega^{*}$ un lacet proche de 0 , d'indice 1 autour de 0 et d'extrémité $x_{0}^{\prime}$. Finalement, choisissons un lacet $\gamma_{0}:[0,1] \rightarrow \Omega^{*}$ d'extrémité $x_{0}^{\prime}$, d'indice 1 autour de $x_{1}$ et d'indice 0 autour de 0 , le long duquel le prolongement analytique de la branche analytique en 0 est remplacée par une autre détermination grâce à la singularité algébrö̈de. Alors, toute détermination de $f(x)$ en $x_{0}$ peut être atteinte par prolongement analytique le long de l'un des chemins $\sigma^{-1} \tau^{-n} \gamma_{0} \tau^{n} \sigma, n \in \mathbb{Z}$. En effet, on a $f_{\tau^{n} \sigma}=f_{\sigma}$ puisqu'il s'agit, de la branche analytique en 0. Par contre, $f_{\gamma_{0} \tau^{n} \sigma}$ est l'une des autres branches près de 0 qui sont transitivement permutées juste en tournant autour de 0, c'est à dire par prolongement analytique le long de chemins $\tau^{-n}$.

Exemple 6.4 (suite de l'exemple 6.2). - Fixons maintenant une verticale $T:\left\{x=x_{0}\right\}$, disons $x_{0}=1$ pour simplifier, et considérons l'application multivaluée $\varphi: T \rightarrow T$; $y \mapsto f\left(x_{0}, x_{0}, y\right)$ construite en recollant tous les germes donnés par le théorème II. Par exemple, considérons $\gamma:[0,1] \rightarrow \Omega=\mathbb{C}^{*}$ un lacet d'indice 1 autour de 0 d'extrémité en $x_{0}$. En appliquant le théorème II à $\gamma$ pour $y_{0}=\infty$ on déduit que le germe d'application d'holonomie $\varphi_{1}$ de la courbe invariante $\{y=\infty\}$ du selle-nœud (voir la remarque 11). L'application multivaluée doit au moins contenir ce germe ainsi que ses itérées $\varphi_{n}:=\varphi_{1}^{\circ n}, n \in \mathbb{Z}$, correspondant à $\gamma^{n}$. Précisément, $\varphi_{1}$ est un germe de difféomorphisme fixant $\infty$ de la forme $\varphi_{1}(y)=y+2 i \pi+\sum_{n>0} \frac{a_{n}}{y^{n}}$. D'autre part, en appliquant le théorème II à $\gamma$ pour $y_{0}=0$ on obtient le germe identité puisque l'holonomie d'une singularité radiale est triviale. 
Bien sûr, le germe identité et $\varphi_{1}$ ne peuvent pas être connectés par prolongement analytique le long du plan des $y$. Donc, l'application multivaluée $\varphi$ doit être la réunion de plusieurs fonctions multiformes. En d'autres termes, son graphe n'est pas irreducible.

Afin d'apréhender l'application $\varphi: y \mapsto f\left(x_{0}, x_{0}, y\right)$ toute entière, remarquons que deux points quelconques $y_{0}$ et $y_{1}$ sur $T$ sont dans la même feuille si et seulement si $H\left(1, y_{0}\right)=H\left(1, y_{1}\right)$. Dans le cas où $y_{0}, y_{1} \neq 0$, ceci signifie encore que $y_{0}+\log \left(y_{0}\right)=y_{1}+\log \left(y_{1}\right)$ pour des déterminations convenables du logarithme. En d'autres termes, $y_{1}=\varphi\left(y_{0}\right)$ pour une détermination de $\varphi$ si et seulement si $\psi\left(y_{0}\right)$ et $\psi\left(y_{1}\right)$ diffèrent d'un élément de $2 i \pi \mathbb{Z}$ (indépendamment de la détermination de $\psi(y)=y+\log (y)$ choisie). Donc, $\varphi$ n'est rien d'autre que le groupe cyclique de translations $2 i \pi \mathbb{Z}$ tiré en arrière par la fonction multiforme $\psi$.

Introduisons le champ de vecteurs $Z=2 i \pi \partial_{z}$ dont l'exponentielle $\exp (Z): z \mapsto z+2 i \pi$ au temps 1 engendre le groupe cyclique précédent. Son relèvement par $\psi$ est $\psi^{*} Z=2 i \pi \frac{y}{1+y} \partial_{y}$. Sa partie réelle est donnée par :

$$
V=\frac{2 \pi}{(1+u)^{2}+v^{2}}\left(\left(u+u^{2}+v^{2}\right) \partial_{v}-v \partial_{u}\right)
$$

où $y=u+i v$. Ainsi, $\varphi$ consiste en la collection de $\exp (n V), n \in \mathbb{Z}$. Ce champ de vecteurs est méromorphe en $y_{0}=-1$ et s'annule en 0 . Au point $y=\infty, \psi^{*} Z$ est holomorphe et $V$ est analytique, tangent au champ de translations $2 \pi \partial_{v}$. Donc, près de l'infini, les trajectoires de $V$ ressemblent à des droites verticales. Le portrait de phases de $V$, donné par le champ de vecteurs polynomial $\left(\frac{(1+u)^{2}+v^{2}}{2 \pi}\right) V=\left(u+u^{2}+v^{2}\right) \partial_{v}-v \partial_{u}$, possède deux singularités, à savoir une selle en $y_{0}$ et un centre en 0 . Les 4 séparatrices de $y_{0}$ consistent en une trajectoire analytique $\sigma^{+}$venant de l'infini depuis le bas et allant vers $y_{0}$, une trajectoire $\sigma^{-}$partant de $y_{0}$ pour aller vers l'infini par le haut et une trajectoire cyclique $\sigma$ se refermant sur $y_{0}$ après avoir tourné une fois autour du centre. Toute autre trajectoire vient de l'infini par le bas pour aller vers l'infini par le haut. Le centre 0 est isochrone pour $V: \exp (V)$ est le germe identité près de 0 . Finalement, introduisons la suite de points $y_{n}$ sur $\sigma^{+} \cup \sigma^{-}, n \in \mathbb{Z}^{*}$, telle que $\exp (n Z)$ envoit $y_{n}$ sur le pôle $y_{0}$. Par exemple, $y_{1}$ est le point limite sur $\sigma^{+}$depuis lequel on peut intégrer $V$ durant le temps 1 . 


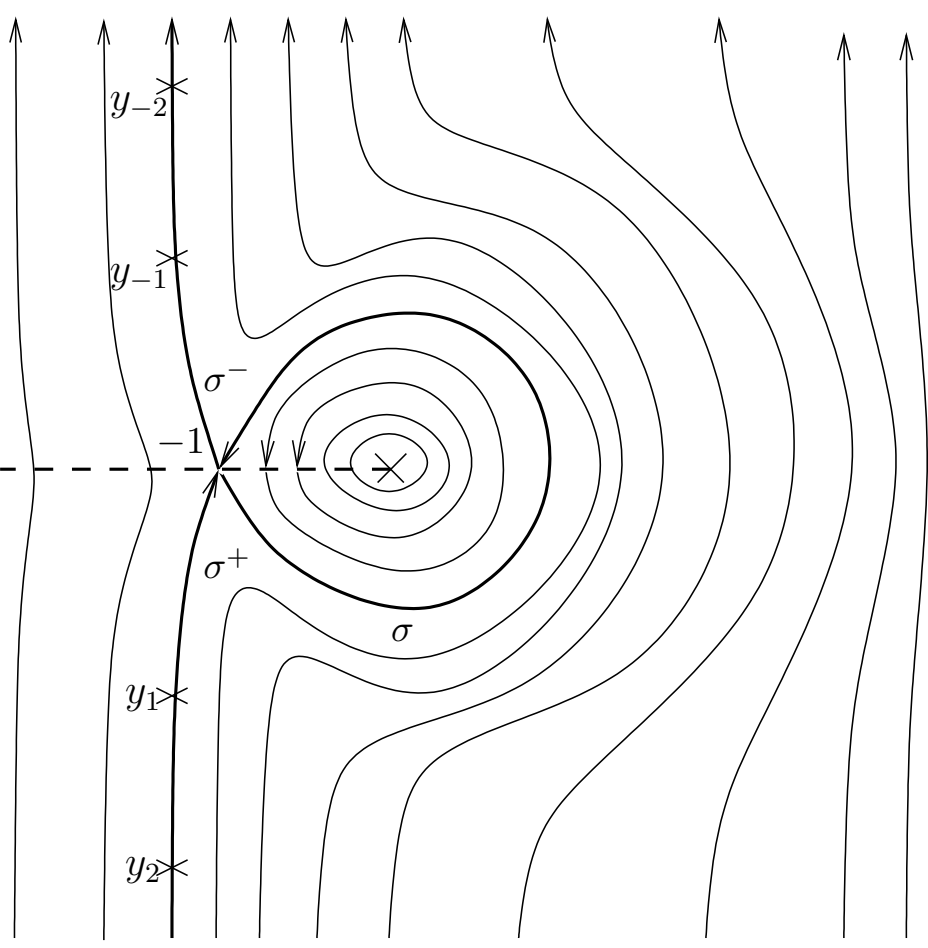

Figure 11. Portrait de phase du champ de vecteurs (réel) $V$

Pour la détermination principale du logarithme, la flèche $\psi: y \mapsto$ $y+\log (y)$ est un homéomorphisme conforme de $\mathbb{C} \backslash \mathbb{R}^{-}$sur $\mathbb{C} \backslash\left(\mathbb{R}^{-}-1 \pm i \pi\right)$. Précisément, $\psi$ envoit difféomorphiquement la frontière :

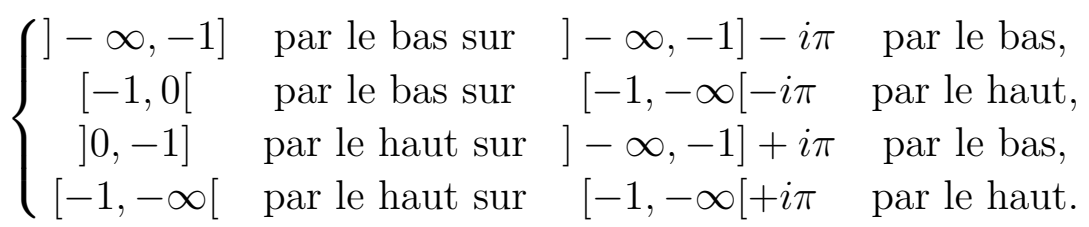

Ceci se voit bien sur l'application $\psi$ écrite en coordonnées polaires :

$$
\psi\left(r e^{i \theta}\right)=[r \cos (\theta)+\log (r)]+i[r \sin (\theta)+\theta] .
$$

Les points $y_{n}$ précédemment définis sont les images réciproques par $\psi$ des points $z_{n}:=-1-(1+2 n) i \pi$ pour $n>0$ et $z_{n}:=-1+(1-2 n) i \pi$ pour $n<0$. Finalement, la monodromie de $\psi$ est $\psi\left(e^{2 i \pi} y\right)=\psi(y)+2 i \pi$.

En raisonnant ou bien sur la dynamique de $V$ ou bien avec l'application $\psi$ telle qu'elle est décrite juste avant, on obtient la description suivante. 


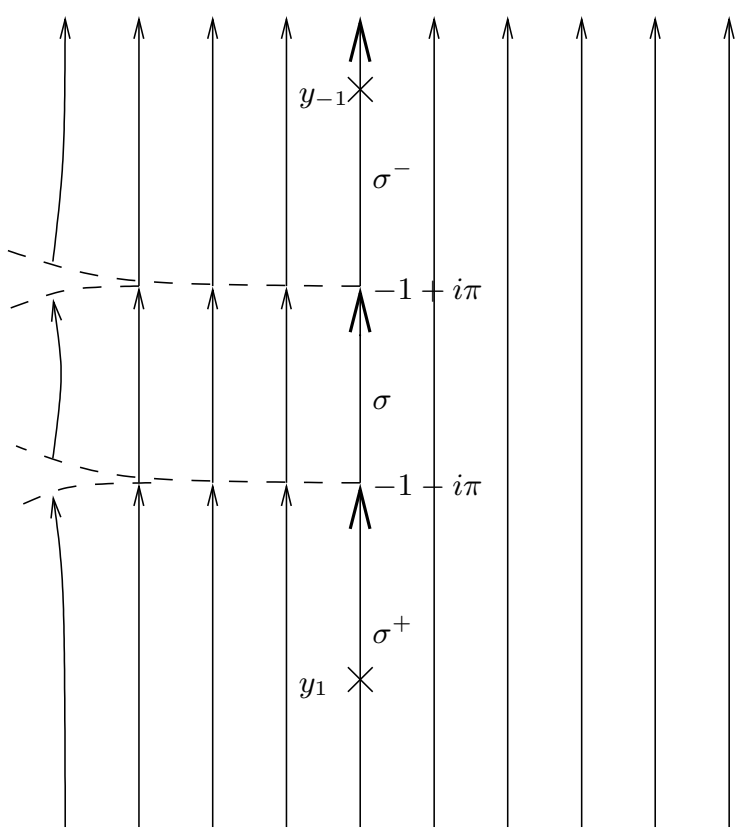

Figure 12. Redressement de $V$ par $\psi$

L'application multivaluée $\varphi$ consiste en exactement deux 2 fonctions, à savoir l'application identité et une fonction multiforme à singularités algébroïdes en $y_{n}, n \in \mathbb{Z}^{*}$, et 2 singularités non algébroïdes en 0 et $\infty$. En particulier, toutes les itérées de l'holonomie $\varphi_{n}, n \in \mathbb{Z}^{*}$, sont les déterminations d'une même fonction. Précisément, pour $n>0, \varphi_{n}$ et $\varphi_{n+1}$ sont permutées autour de $y_{n}$ et $\varphi_{-n}$ et $\varphi_{-n-1}$ sont permutées autour de $y_{-n}$. Les points $y_{n}$ sont effectivement singuliers uniquement lorsqu'ils sont vus depuis ces branches. Le point $\infty$ est régulier pour toutes les déterminations mais devient singulier dans la situation suivante. Partons d'une branche $\varphi_{1}$ et procédons au prolongement analytique le long d'une trajectoire $\sigma^{+}$jusqu'à $y_{1}$. Alors, changeons de détermination autour de $y_{1}$ et revenons au point $\infty$ le long de $\sigma^{+}$en tournant successivement une fois autour de chacun des points $y_{2}, y_{3}, y_{4}, \ldots$ dans cet ordre. En particulier, $\varphi$ n'a pas de limite le long de ce chemin puisque $\varphi(y)$ parcours $\sigma$ entre deux singularités successives intermédiaires $y_{n}, y_{n+1}$. Par ailleurs, toutes les branches ont pour limite $\infty$ en 0 et se permutent transitivement autour, comme pour la fonction logarithme. 
Les singularités algébroïdes $y_{n}, n \in \mathbb{Z}^{*}$, sont les points d'intersection de la verticale $T$ avec la feuille passant par $(1,-1)$, c'est à dire qui est tangente à $T$. Elles apparaissent lorsque, partant d'une solution analytique en $\left(1, y_{n}\right)$, on procède au prolongement analytique le long d'un chemin $\gamma$ tournant $n$ fois autour de 0 (dans le plan des $x$ ) et revenant au point 1 avec la solution algébrö̈de en $(1,-1)$, i.e. avec tangence sur $T$.

\section{Conjectures}

Le théorème I ainsi que les exemples précédents décrivent complètement le type de singularités des solutions et la façon dont elles apparaîssent. Le théorème II, quant à lui, ne nous fournit pas d'indication sur le comportement des applications d'holonomie vis à vis du prolongement analytique. L'exemple 6.1 nous montre d'ores et déjà que ces applications ne sont pas globalement algébroïdes : des singularités plus compliquées peuvent apparaître. Nous proposons deux conjectures.

Conjecture 1. - Soient $P, Q \in \mathbb{C}[x, y]$ et considérons l'équation différentielle :

$$
\text { (E) } \quad \frac{d y}{d x}=\frac{P(x, y)}{Q(x, y)}
$$

Supposons que ni $P$, ni $Q$, ne soient identiquement nuls et considérons deux droites verticales $T_{0}$ et $T_{1}$ (non invariantes par le feuilletage) ainsi qu'un germe d'application algébroide $\varphi:\left(T_{0}, p_{0}\right) \rightarrow\left(T_{1}, p_{1}\right)$ donné par le Théorème II, $p_{i} \in T_{i}, i=0,1$. Alors l'ensemble $\Sigma_{\varphi}$ des singularités de $\varphi$ pour le prolongement analytique dans $T_{0} \simeq \overline{\mathbb{C}}$ est au plus dénombrable.

En particulier, $\varphi$ admet un prolongement sans coupure : le phénomème de l'exemple 2.8 ne se produit pas. Cette conjecture fut motivée au départ par le problème suivant. Si l'énoncé est vrai, alors on peut construire une intégrale première multiforme pour le feuilletage dont l'ensemble singulier est une réunion dénombrable de feuilles. Une telle fonction admet automatiquement un groupe de monodromie (voir [6]) qui sera en général très consistant. À ma connaissance, on n'a jamais associé que des pseudo-groupes à un feuilletage algébrique général, ce qui rend difficile la construction d'une théorie de Galois pour ce type d'objets. 
Dans le cas où les singularités de l'équation (E) sont toutes hyperboliques, il est vraisemblable que les singularités non algébroïdes de $\varphi$ naissent des applications de Dulac locales des singularités. On peut alors s'attendre à ce qu'elles se situent sur les points d'intersection de la droite $T_{0}$ avec les feuilles (en nombre fini) "passant par ces singularités" sous forme de courbes invariantes locales. Notamment, l'application $\varphi$, après prolongement analytique maximal, mettrait en relation des points de $T_{0}$ et $T_{1}$ qui ou bien seraient dans la même feuille, ou bien seraient dans des feuilles distinctes se connectant comme courbes invariantes locales aux singularités (comme un polycycle). C'est en tout cas ce qui se passe dans tous les exemples étudiés. Le lecteur pourra trouver, dans la thèse de David Marin (voir [7]), d'autres exemples instructifs illustrant cette conjecture.

Cependant, dans tous les exemples étudiés, on utilise une intégrale première multiforme explicite du feuilletage (que l'on inverse) pour pouvoir obtenir une description globale de $\varphi$. Nous ne sommes pas capable actuellement de donner un seul exemple d'équation non intégrable (explicitement) satisfaisant notre conjecture. Ces applications $\varphi$ peuvent être extrèmement compliquées. Par exemple, un travail récent (voir [1]) montre que ces applications ne satisfont généralement aucune équation différentielle analytique et sont, en ce sens, plus transcendantes que toutes les fonctions solutions de toutes les équations différentielles. Dans cette direction, nous proposons cette autre :

Conjecture 2. - Soient $P, Q \in \mathbb{C}[x, y]$ et considérons l'équation différentielle :

$$
\text { (E) } \frac{d y}{d x}=\frac{P(x, y)}{Q(x, y)} .
$$

Supposons que ni $P$, ni $Q$, ne soient identiquement nuls et que $Q$ ne contient aucun facteur vertical : aucune droite verticale n'est totalement tangente au feuilletage. Considérons alors une droite verticale $T_{0}$ et deux germes d'applications analytiques $\varphi:\left(T_{0}, p_{0}\right) \rightarrow\left(T_{0}, p_{1}\right)$ et $\varphi^{\prime}:\left(T_{0}, p_{0}\right) \rightarrow$ $\left(T_{0}, p_{1}^{\prime}\right)$ donnés par le Théorème II. Alors $\varphi^{\prime}$ se déduit par prolongement analytique de $\varphi$ le long de $T_{0} \ldots$ sauf si l'un des deux germes est l'identité. 
Une conséquence immédiate de la conjecture 2 et de [1] serait :

si $P$ et $Q$ sont suffisamment génériques de degré $d \geq 2$, alors le prolongement analytique maximal de $\varphi$ accumule tout le pseudo-groupe des transformations conformes sur $T_{0}$ : pour tout germe d'application analytique inversible $\psi:\left(T_{0}, p_{0}\right) \rightarrow T_{1}$, il existe une suite de déterminations $\varphi_{n}:\left(T_{0}, p_{0}\right) \rightarrow T_{1}$ de $\varphi$ en $p_{0}$ convergeant uniformément vers $\psi$ sur un voisinage de $p_{0}$ dans $T_{0}$.

Il serait joli de construire un tel exemple.

\section{Références}

[1] M. Belliart, I. Liousse et F. Loray, The generic rational differential equation $\frac{d w}{d z}=\frac{P_{n}(z, w)}{Q_{n}(z, w)}$ on $\mathbb{C P}^{2}$ carries no interesting transverse structure. Ergodic Theory et Dyn. Syst. 21 (2001) 1599-1607.

[2] P. Boutroux, Leçons sur les fonctions définies par les équations différentielles du premier ordre. Gauthier-Villars Paris, 1908.

[3] R. Gérard et A. Sec, Feuilletages de Painlevé. Bull. Soc. math. France 100 (1972) p.47-72.

[4] M. Hukuhara, T. Kimura, T. Matuda, Équations différentielles ordinaires du premier ordre dans le champ complexe. Publications of the Mathematical Society of Japan, 7, Tokyo, 1961.

[5] Jouanolou, Équations de Pfaff algébriques. Lecture Notes in Mathematics, 708, Springer Berlin, 1979.

[6] A. Khovanskii, Topological obstructions to the representability of functions by quadratures. J. Dynam. Control Systems 1 (1995) 91-123.

[7] D. Marín Pérez, Problemas de módulos para una clase de foliaciones holomorfas. Thèse de l'Université Autonôme de Barcelone, 2000.

[8] P. Painlevé, CEuvres complètes. Éditions du C.N.R.S. (R. Gérard, G. Reeb et A. Sec) Paris, 1972.

[9] G. Reeb, Feuilletages. Résultats anciens et nouveaux (Painlevé, Hector et Martinet). Les presses de l'Université de Montréal, 1974.

[10] G. Valiron, Théorie des fonctions. Masson et Cie, Paris, 1942.

[11] H. Weyl, The concept of a Riemann surface. Traduit de la troisième édition allemande par Gerald R. MacLane. ADIWES International Series in Mathematics, Addison-Wesley Publishing Co., Inc., Reading, Mass. London, 1964.

February 2004 
Frank LORAY, (Chargé de Recherches au CNRS), IRMAR, UFR de Mathématiques,, Université de Rennes 1, Campus de Beaulieu,, 35042 Rennes Cedex (France) - E-mail : frank.loray@univ-rennes1.fr Url : http://name.math.univ-rennes1.fr/frank. loray 